Mon. Not. R. Astron. Soc. 000,1 20(2010) Printed 10 October $2018 \quad$ (MN LATEX style file v2.2)

\title{
The glitch-induced identity changes of PSR J1119-6127
}

\author{
Patrick Weltevrede ${ }^{1,2 \star}$, Simon Johnston ${ }^{2}$ and Cristóbal M. Espinoza ${ }^{1}$ \\ ${ }^{1}$ Jodrell Bank Centre for Astrophysics, The University of Manchester, Alan Turing Building, Manchester, M13 9PL, United Kingdom. \\ ${ }^{2}$ Australia Telescope National Facility, CSIRO, P.O. Box 76, Epping, NSW 1710, Australia.
}

\begin{abstract}
Rotation-powered radio pulsars are generally observed to pulse regularly in the radio band, but this is not the case for so-called rotating radio transients (RRATs) which emit only sporadic bursts of radio emission. We demonstrate that the high-magnetic field pulsar J1119-6127 exhibits three different types of behaviour in the radio band. Trailing the "normal" profile peak there is an "intermittent" peak and these components are flanked by two additional components showing very erratic "RRAT-like" emission. Both the intermittent and RRAT-like events are extremely rare and are preceded by a large amplitude glitch in the spin-down parameters. The post-glitch relaxation occurs on two different timescales ( $\sim 20$ and $\sim 210$ days $)$ and the post-glitch spin-down rate is smaller than the pre-glitch rate. This type of relaxation is also seen in an earlier, smaller glitch and is very unusual for the pulsar population as a whole, but is observed in the glitch recovery of a RRAT. The abnormal emission behaviour in PSR J1119-6127 was observed up to three months after the epoch of the large glitch, suggestive of changes in the magnetospheric conditions during the fast part of the recovery process. We argue that both the anomalous recoveries and the emission changes could be related to reconfigurations of the magnetic field. Apart from the glitches, the spin-down of PSR J1119-6127 is relatively stable, allowing us to refine the measurement of the braking index $(n=2.684 \pm 0.002)$ using more than 12 years of timing data.

The properties of this pulsar are discussed in light of the growing evidence that RRATs do not form a distinct class of pulsar, but rather are a combination of different extreme emission types seen in other neutron stars. Different sub-classes of the RRATs can potentially be separated by calculating the lower limit on the modulation index of their emission. Unlike other quantities, this parameter is independent of observation duration allowing a direct comparison with other emission phenomenon. We speculate that if the abnormal behaviour in PSR J1119-6127 is indeed glitch induced then there might exist a population of neutron stars which only become visible in the radio band for a short duration in the immediate aftermath of glitch activity. These neutron stars will be visible in the radio band as sources that only emit some clustered pulses every so many years.
\end{abstract}

Key words: pulsars: individual: J1119-6127 — pulsars: general — radiation mechanisms - polarization

\section{INTRODUCTION}

PSR J1119-6127 is a young, isolated radio pulsar discovered by Camilo et al. (2000) with the Parkes radio telescope in Australia. Its period $(P=0.41 \mathrm{~s})$ has a typical value observed for pulsars, but its period derivative $\left(\dot{P}=4.0 \times 10^{-12}\right)$ is among the highest known. Therefore the inferred surface (dipole) magnetic field strength is very large $\left(B_{\mathrm{S}}=4.1 \times 10^{13}\right.$
G), making this pulsar one of the "high-magnetic field pulsars". This field strength is comparable to the so-called "quantum critical field strength" of $B_{\mathrm{cr}}=4.4 \times 10^{13} \mathrm{G} 1$ above which photon splitting may prevent pair production (Baring \& Harding 1998). Because this pulsar does not rotate particularly rapidly, the magnetic field strength at the

\footnotetext{
* E-mail: Patrick.Weltevrede@manchester.ac.uk
} 
light cylinder $\left(B_{\mathrm{LC}}=5.7 \times 10^{3} \mathrm{G}\right)$ is high, but not as extreme as that of other pulsars. From its spin parameters one can further infer its characteristic age $\left(\tau_{c}=1.6 \mathrm{kyr}\right)$, which makes its association with supernova remnant G292.2-0.5 likely (Crawford et al. 2001).

The high spin-down energy loss rate $\left(\dot{E}=2.3 \times 10^{36}\right.$ $\mathrm{erg} \mathrm{s}^{-1}$ ) makes this source a promising target for the Fermi $\gamma$-ray satellite (Smith et al. 2008). Hence it is one of the energetic pulsars which are currently monitored using the Parkes radio telescope allowing the detection of possible $\gamma$ ray pulsations (Weltevrede et al. 2010). During these (ongoing) observations we noted that this pulsar has striking similarities with a group of neutron stars called rotating radio transients (RRATs; McLaughlin et al. 2006) and with the intermittent pulsars (PSR B1931+24 being the first discovered; Kramer et al. 2006). This pulsar can therefore be seen, like PSR B0656+14 (Weltevrede et al. 2006), as an object that links different "classes" of neutron stars. We briefly introduce these different types of objects below.

RRATs are characterized by the sporadic nature of their emission. Typically, detectable radio emission is observable for less than one second per day, causing standard periodicity searches to fail in detecting a periodic signal. Therefore one has to rely on the greatest common divisor of the time between bursts to derive the underlying rotational period of the star. There are about forty reported RRATs in the literature (McLaughlin et al. 2006; Hessels et al. 2008; Deneva et al. 2009; Keane et al. 2010; Burke-Spolaor \& Bailes 2010). Many RRATs have relatively long periods up to $7 \mathrm{~s}$, suggesting these sources may be related to the radio-quiet X-ray populations of neutron stars, such as magnetars (Woods \& Thompson 2006) and isolated neutron stars (Kaplan 2008), which are observed to have similar periods. The size of the RRAT population is thought to be several times larger than the radio pulsar population (McLaughlin et al. 2006), leading to inconsistencies with the observed supernova rate unless there is an evolutionary link between the neutron star classes (Keane \& Kramer 2008). Different ideas are put forward to explain the sporadic nature of detectable pulses, such as intermittent particle precipitation towards the star from a radiation belt similar to those in planetary magnetospheres (Luo \& Melrose 2007) or re-activation of the usually inactive vacuum gap due to in falling circumstellar asteroidal material (Cordes \& Shannon 2008).

Weltevrede et al. (2006) argue that PSR B0656+14 would have been classified as a RRAT were it not one of the most nearby pulsars. One is therefore left with the question if PSR B0656+14 should be called a RRAT? If not, it implies that at least some of the "RRATs" are physically very similar to PSR B0656+14, except that they are more distant. In any case, it is clear that defining RRATs to be pulsars that are not detectable via periodicity searches is insufficient and that one has to define the different neutron star classes based on physical properties instead (e.g. Keane et al. 2010).

Based on the similarities between PSR B0656+14 and the RRATs, Weltevrede et al. (2006) predicted that at least some of the RRATs would show additional emission which is much weaker than their bright individual pulses and that when the individual bursts of RRATs are averaged they will form a "profile" much broader than the individ- ual bursts. Indeed, for instance the RRAT PSR J0627+16, which was found via its individual pulses, showed weak emission in a follow-up observation (Deneva et al. 2009) and the sum of the individual bursts of the RRAT PSR J18191458 produces a triple peaked pulse profile which is much wider than the individual bursts (Karastergiou et al. 2009). The latter RRAT shows a X-ray spectrum which is consistent with thermal emission from a cooling neutron star (Reynolds et al. 2006; Gaensler et al. 2007) and, like PSR B0656+14, it shows X-ray pulsations (McLaughlin et al. 2007). As will be discussed later in this paper, the peculiar emission properties of PSRs J1119-6127 and B0656+14 might be linked to the erratic emission seen for other young and energetic pulsars, such as the Vela pulsar and PSR B1706-44 (Johnston et al. 2001; Johnston \& Romani 2002), thereby providing some generalization between the different phenomenon.

The final class of neutron stars we will discuss in the introduction are the intermittent pulsars. The archetype is PSR B1931+24 (Kramer et al. 2006), which is only active for a few days between periods of roughly a month during which the pulsar is not detectable. These sudden switches are accompanied by changes in the spin-down rate of the neutron star rotation, suggestive of significant changes in the torque generated by magnetospheric currents. It now has become clear that many more objects show pulse profile changes concurrent with changes in the spin-down rate (Lyne et al. 2010) and it has been suggested that all the so-called timing-noise could be ascribed to this effect. Indeed the spin parameters of PSR B1931+24 $(P=0.81 \mathrm{~s}$ and $\dot{P}=8.1 \times 10^{-15}$ ) and other physical quantities such as $B_{\mathrm{S}}=2.6 \times 10^{12} \mathrm{G}, \dot{E}=5.9 \times 10^{32} \mathrm{erg} \mathrm{s}^{-1}$ and $\tau_{c}=1.6 \mathrm{Myr}$ are not remarkable, supporting the idea that the correlated profile and spin-down switches could be very common in the pulsar population. It is therefore natural to extend this idea to all pulsars that switch off (or change their profile) on a regular basis during so-called "nulls" or "mode changes". The nulls of some pulsars are very short (a period or less), while others are only switched on for a few percent of the time (e.g. Wang et al. 2007).

In the next sections we give some details about the observations (Sect. 2) and we will describe a transient profile change in Sect. 3. This is followed by the analysis of the individual pulses (Sect. 4), polarization (Sect. 5) and pulsar timing behaviour (Sect. 6). The paper ends with a discussion of the properties of PSR J1119-6127 in the light of the different neutron star classes (Sect. 7) and the conclusions (Sect. 8).

\section{OBSERVATIONS}

All the discussed observations were carried-out using the 64$\mathrm{m}$ Parkes radio telescope in Australia and the majority of the data were obtained as part of the aforementioned timing program (Weltevrede et al. 2010). This program started in April 2007. Each pulsar is typically observed once per month at $20 \mathrm{~cm}$ and twice per year at 10 and $50 \mathrm{~cm}$, although this pulsar is only detected at 10 and $20 \mathrm{~cm}$. The used receivers were the centre beam of the $20 \mathrm{~cm}$ multibeam receiver (which has a bandwidth of $256 \mathrm{MHz}$ and has a noise equivalent flux density of $\sim 35$ Jy on a cold sky) 

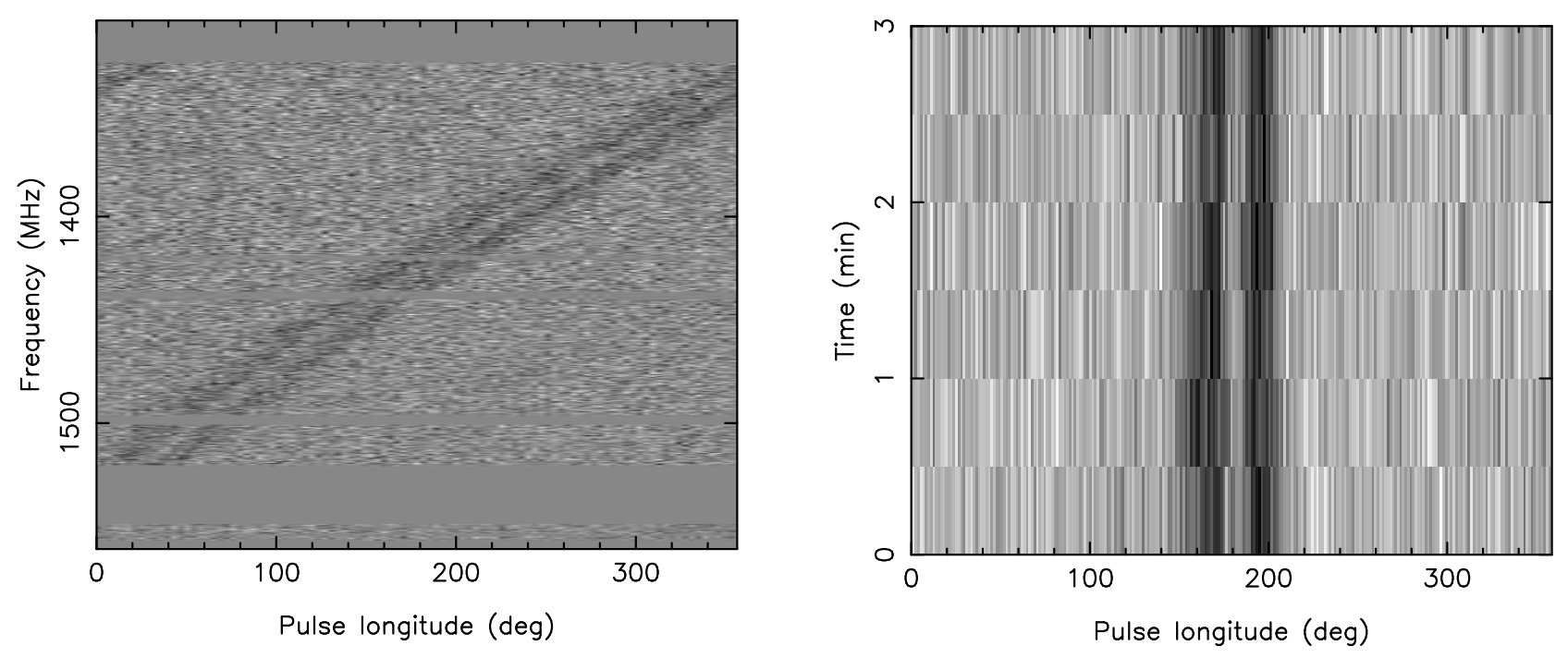

Figure 1. The only observation known to us showing PSR J1119-6127 in its double-peaked mode. In both panels the intensity is shown in grayscale. Left-hand panel: Both profile components are identically affected by dispersion (an observing frequency dependent delay caused by the interstellar medium), resulting in the two parallel diagonal bands in this panel. Right-hand panel: The double-peaked pulse profile was visible during the entire 3 minute observation.

and the $10 / 50 \mathrm{~cm}$ receiver (which has at $10 \mathrm{~cm}$ a bandwidth of $1024 \mathrm{MHz}$ and has a noise equivalent flux density of $\sim 49 \mathrm{Jy}$ on a cold sky). The data were on-line folded at the pulse period by a digital filterbank and were dumped every 30 seconds on hard disk. For details about the polarization calibration procedure and the used method to sum all the individual observations into a high signal-to-noise "standard" profile we refer to Weltevrede \& Johnston (2008). For the majority of the observations the analogue filterbank was used to record the pulsar signal in parallel with a time resolution of $250 \mu \mathrm{s}$. Although only total intensity is recorded it has the advantage that the data are not folded on-line, hence allowing the analysis of individual pulses.

In addition we made use of archival Parkes data recorded between 1998 February 7 and 2007 February 12. For details about these observations we refer to Camilo et al. (2000) in which some of these analogue filterbank data are published. Together with data from our timing program up to 2010 June 16 , this results in a timing history of more than 12 years.

\section{THE TRANSIENT PROFILE COMPONENT}

The pulse profile of PSR J1119-6127 is well known to be single-peaked (see e.g. Camilo et al. 2000; Johnston \& Weisberg 2006). The observation recorded in 2007 June 17 at 02:35:19 Universal Time therefore immediately triggered our interest in this object as it shows a clear double-peaked profile (see Fig. 11). Despite years of trying we never observed a similar event again. Hence, it was important to convince ourselves that the signal was pulsar related rather than caused by something instrumental or by terrestrial radio frequency interference (RFI). As one can see in the left panel of Fig. 1 both peaks of the pulse profile show an identical frequency dependent delay (the gray horizontal bands are frequency channels affected by RFI and were excluded from the analysis). This frequency dependent delay is exactly the same as what is normally observed for this pulsar and can be perfectly explained in terms of dispersion by the interstellar medium. This, combined with the fact that the double-peaked structure is seen during the whole observation after folding at the known pulse period (see right panel of Fig. 1) rules out RFI as the cause of the additional component. The signal was recorded using two independent digital filterbank backend $\$^{2}$ and the recordings look identical as expected. All observations before and after this particular observation (of different pulsars) used an identical system set-up and do not show any sign of a similar effect. Therefore the appearance of the additional component is clearly pulsar related.

With only one instance of the pulsar showing its doublepeaked mode and no observation of an actual transition it is impossible to reliably estimate the occurrence rate of this transient phenomenon. The pulsar was double peaked during the whole 3 minutes of the observation and the observations 49 days earlier and 35 days later look normal, implying a total duration of the event of at least minutes, but shorter than months. In order to maximize the chance of catching the pulsar again during a similar event we substantially increased the observing duration (up to half an hour) in subsequent observing sessions. In total we have observed this pulsar 76 times in between 2007 April 30 and 2010 June 16, typically twice a day every month with a total duration of 7.9 hours. In addition we inspected archival Parkes data recorded between 1998 February 7 and 2007 February 12. None of these 256 additional observations (with a total duration of 41.9 hours) shows a similar event. Therefore the double-peaked mode is clearly very rare as it is only seen in $0.10 \%$ of the inspected data.

\footnotetext{
2 The used backends are known as "DFB1" and "DFB2".
} 


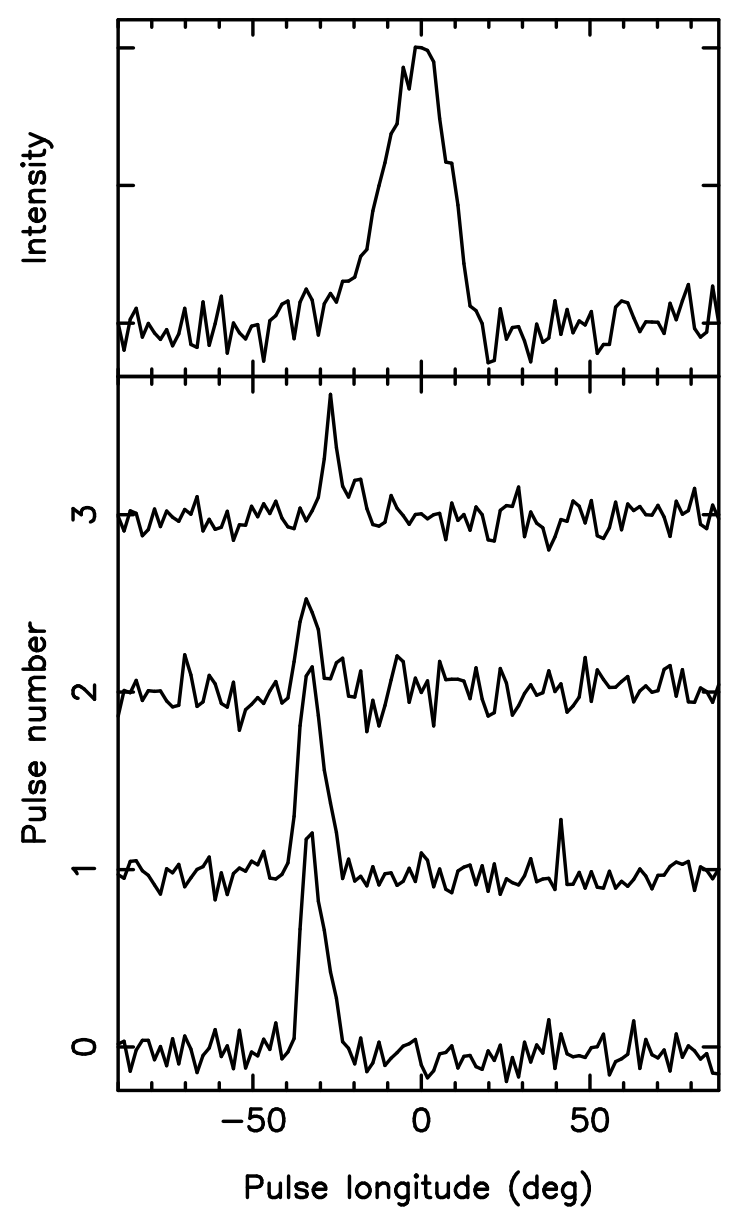

Figure 2. Top panel: Averaged pulse profile of a single $22 \mathrm{~min}-$ utes long observation at a wavelength of $20 \mathrm{~cm}$. The time resolution is reduced to 200 bins across the pulse period and the peak of the profile is put at pulse longitude $0^{\circ}$. Bottom panel: The strong (non-consecutive) individual pulses observed during the same observation. Notice that at the pulse longitude of the bright individual pulses there is no integrated power visible, which is reminiscent of the emission of RRATs.

\section{SINGLE-PULSE ANALYSIS: THE RRAT-LIKE COMPONENTS}

\section{$4.1 \quad 20-\mathrm{cm}$ observations}

At first glance this pulsar is not a promising source to analyse its individual pulses because it is quite weak (the average single-pulse signal-to-noise is less than 1). Nevertheless, careful investigation by eye of all the observations for which we had single-pulse recordings available revealed a handful of strong pulses. In total only four pulses at an observing wavelength of $20 \mathrm{~cm}$ were strong enough to be clearly detectable (see Fig. 2). They all occurred during a 22 minutes long observation recorded at 2007 August 20, starting at 22:11:05 UT.

Strong pulses were visible only in one of the 217 observations (with a total duration of 28.3 hours) for which we have single-pulse data (and which were not too badly affected by RFI). In other words, only during 4 of the 246,892 inspected neutron star rotations the intensity of the radio beam was strong enough to be detectable with the Parkes telescope.
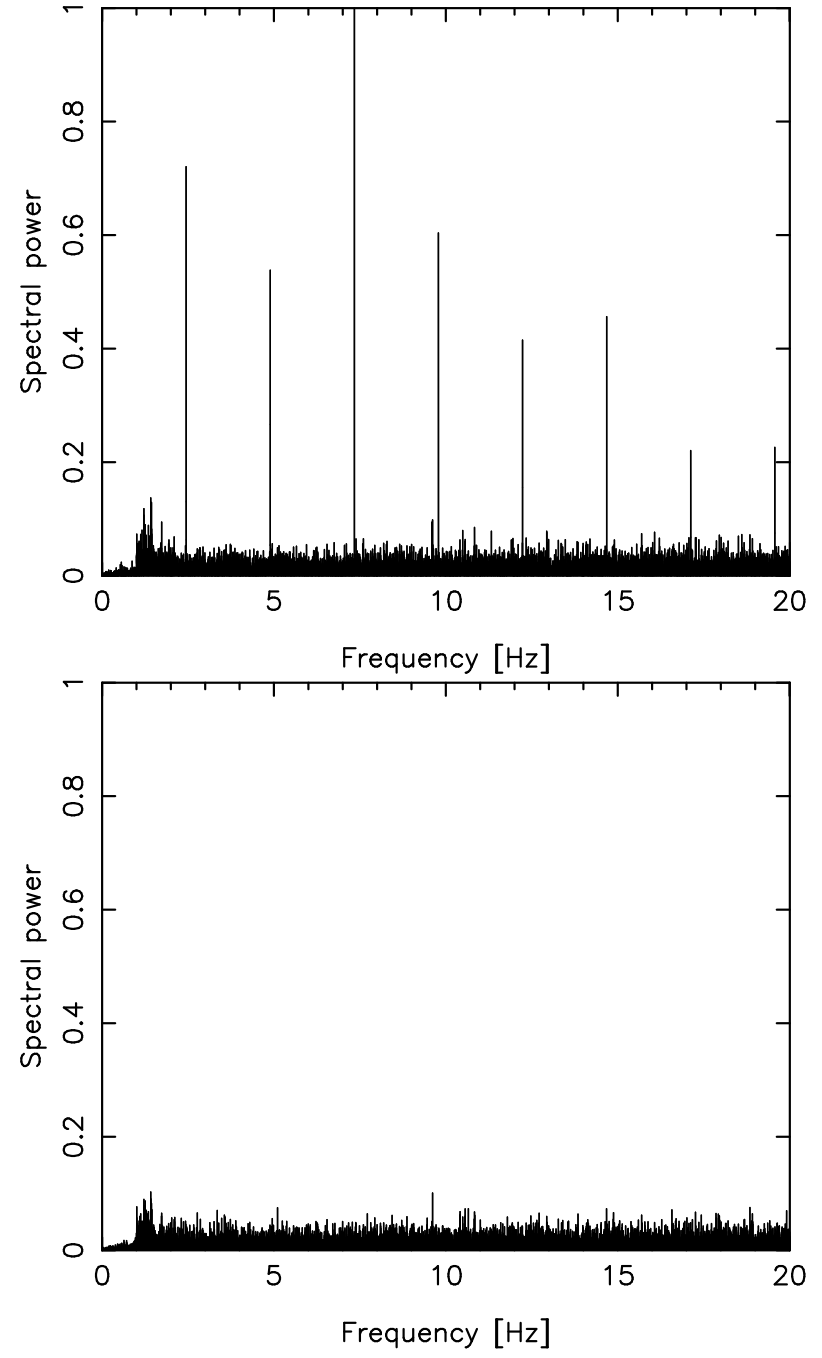

Figure 3. Top panel: Spectrum of the time-series of the observation containing the four strong pulses shown in Fig. 2 The periodicity of the pulsar signal clearly stands out as a spike at $2.45 \mathrm{~Hz}$ and spikes at higher frequencies harmonically related to the fundamental frequency. Bottom panel: The spectrum of the same time-series after replacing the signal at the pulse longitudes corresponding to the normal emission by Gaussian noise thereby only keeping the strong pulses. None of the visible spikes correspond to the periodicity of the pulsar. The normalization of the spectral power is the same in both panels. The decrease in spectral power below $\sim 1 \mathrm{~Hz}$ is due to a software-filter which removes baseline variations.

Assuming a constant event rate for the occurrence of strong pulses, Poisson statistics predicts an extremely low probability of $3 \times 10^{-7}$ that four (or more) pulses occur during a single 3211 pulse period long observation by pure chance. This strongly suggests that the event rate must have been much higher during that particular observation.

Looking at Fig. 2, one can see that the strong individual pulses all occur $\sim 35^{\circ}$ in pulse longitude earlier than the peak of the normal profile. Surprisingly, at the pulse longitudes one can detect individual pulses, the integrated profile does not show significant emission. This is a defining characteristic of RRATs, as it implies that this kind of emission cannot be detected using standard periodicity searches. 
To prove this point, we took the observation with the four strong pulses and replaced the emission within the pulse longitude range corresponding to the normal emission with Gaussian noise with a root mean square (RMS) equal to the off-pulse RMS. The spectrum based on this time-series is shown in the bottom panel of Fig. 3. while the spectrum including the normal emission is shown in the top panel. One can clearly see that the fundamental frequency $(2.45 \mathrm{~Hz})$ and the higher harmonics are only visible when the normal emission is included. Despite the strongest pulses are observed in the leading component, the underlying periodicity (or the underlying emission itself) is not detectable at those pulse longitudes. Therefore the leading component can be qualified to have RRAT-like emission, although as will be discussed later, there are also important differences.

The strongest of the four detected pulses is 23 times brighter than the average pulse of the normal emission during the same observation. Assuming the normal emission of the pulsar was not abnormally bright or weak during that particular observation (and there is no reason to believe so based on the signal-to-noise ratio of the pulse profile of that observation compared to others), this corresponds to $18 \mathrm{mJy}$ averaged out over a whole pulse period (using the published mean flux density of $0.80 \mathrm{mJy}$ at $1400 \mathrm{MHz}$ by Manchester et al. 2001) or a peak flux density of 0.6 Jy using the full single-pulse width of $11^{\circ}$.

To investigate how "normal" the radio emission in the main-peak following the RRAT-like component of PSR J1119-6127 is, we attempted to calculate the modulation index for this pulsar. The modulation index is independent of the observing system and quantifies the broadness of an amplitude distribution. It is defined to be

$m=\frac{\sigma_{I}}{\langle I\rangle}=\left(\frac{1}{N_{\text {tot }}} \sum_{i=1}^{N_{\text {tot }}}\left(I_{i} /\langle I\rangle-1\right)^{2}\right)^{1 / 2}$

where $\sigma_{I}$ is the standard deviation of the observed pulse intensities, $\langle I\rangle$ is the average pulse intensity and $N_{\text {tot }}$ is the total number of stellar rotations observed. This quantity can be calculated as function of pulse longitude by considering the intensities at a specific longitude rather than the pulse intensities integrated over pulse longitude. We have chosen to calculate the modulation index in the Fourier domain instead, which as explained by Edwards \& Stappers (2002) allows a correction for interstellar scintillation.

It turned out that the signal-to-noise ratio in an individual observation is not high enough to detect a significant modulation index. To circumvent this problem we have added together all the individual pulse recording at a wavelength of $20 \mathrm{~cm}$. In order to do this the average off-pulse intensity of each observation was subtracted, the data were re-sampled to have an equal number of bins across the pulse period and the intensities were scaled to make the off-pulse RMS equal to 1 (thereby assuming that the system temperature was the same for all observations). The individual observations were then aligned by cross-correlating their pulse profiles with an analytic template based on von Mises functions (see e.g. Weltevrede \& Johnston 2008). The resulting pulse stack is based on more than 28 hours of data and shows a modulation index of $m \sim 0.5$ in the centre of the profile, flaring up at the edges. It is likely that the modulation index is slightly lower in reality because the addition process can

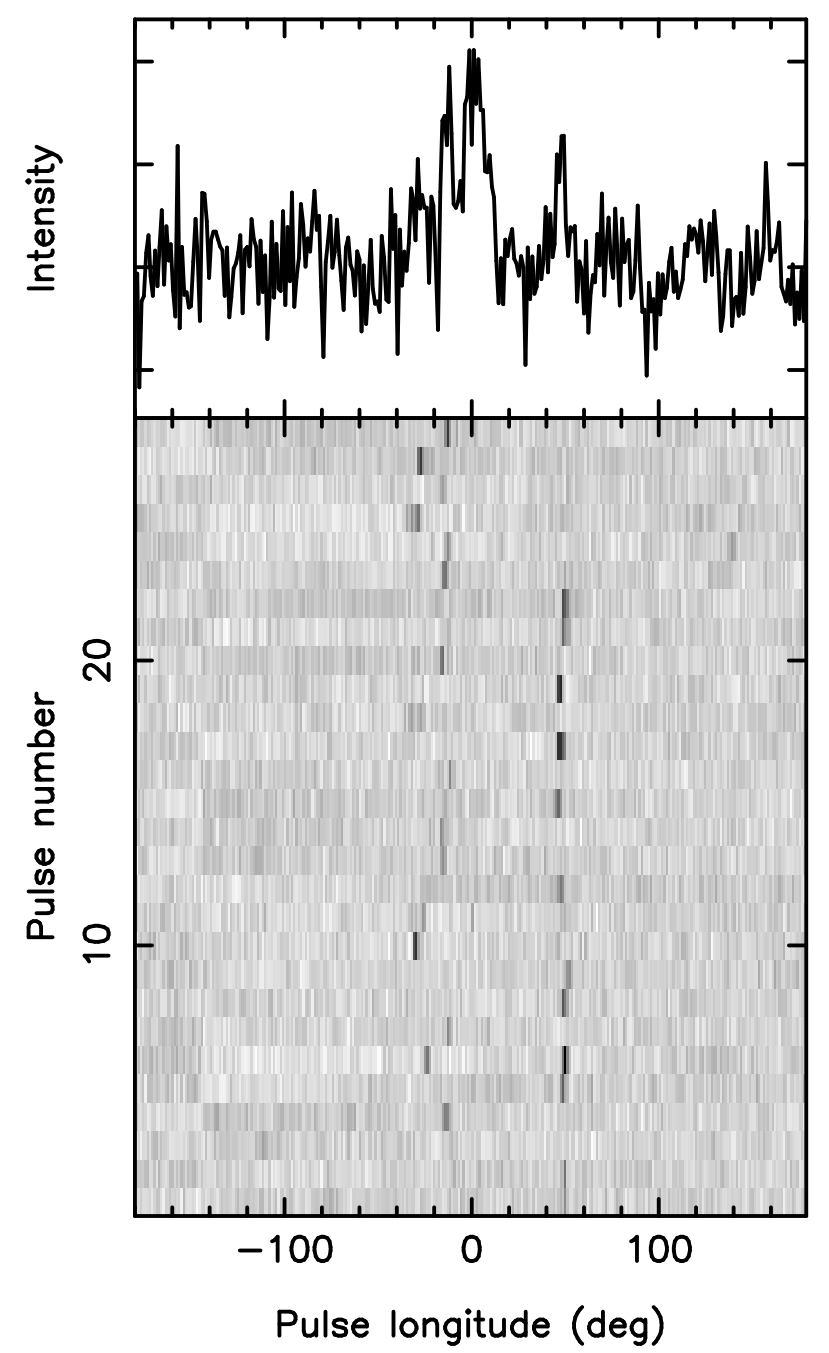

Figure 4. Top panel: Pulse profile of a single 6 minute observation at a wavelength of $10 \mathrm{~cm}$. The time resolution is reduced to 300 bins across the pulse period. The peak of the pulse profile is placed at zero longitude. Bottom panel: The strong (nonconsecutive) individual pulses observed in the same six minute observation are plotted in grayscale.

add some artificial variations to the signal. Nevertheless, the modulation index of PSR J1119-6127 is entirely consistent to those observed for most pulsars (e.g. Weltevrede et al. 2006).

\section{$4.2 \quad 10-c m$ observations}

PSR J1119-6127 has only been observed sporadically at a wavelength of $10 \mathrm{~cm}$ during our timing program and at a wavelength of $50 \mathrm{~cm}$ the pulsar is too weak to be detectable for the used integration times. It must be noted that the combination of a high dispersion measure, worse system temperature, smaller band width and a much higher sky temperature might well be preventing us from detecting the pulsar at $50 \mathrm{~cm}$. Because the individual pulses were not always recorded at those wavelengths, there are only two individual pulse recordings available at $10 \mathrm{~cm}$. One of these recordings, the 6 minute long 2007 July 23 observation, 


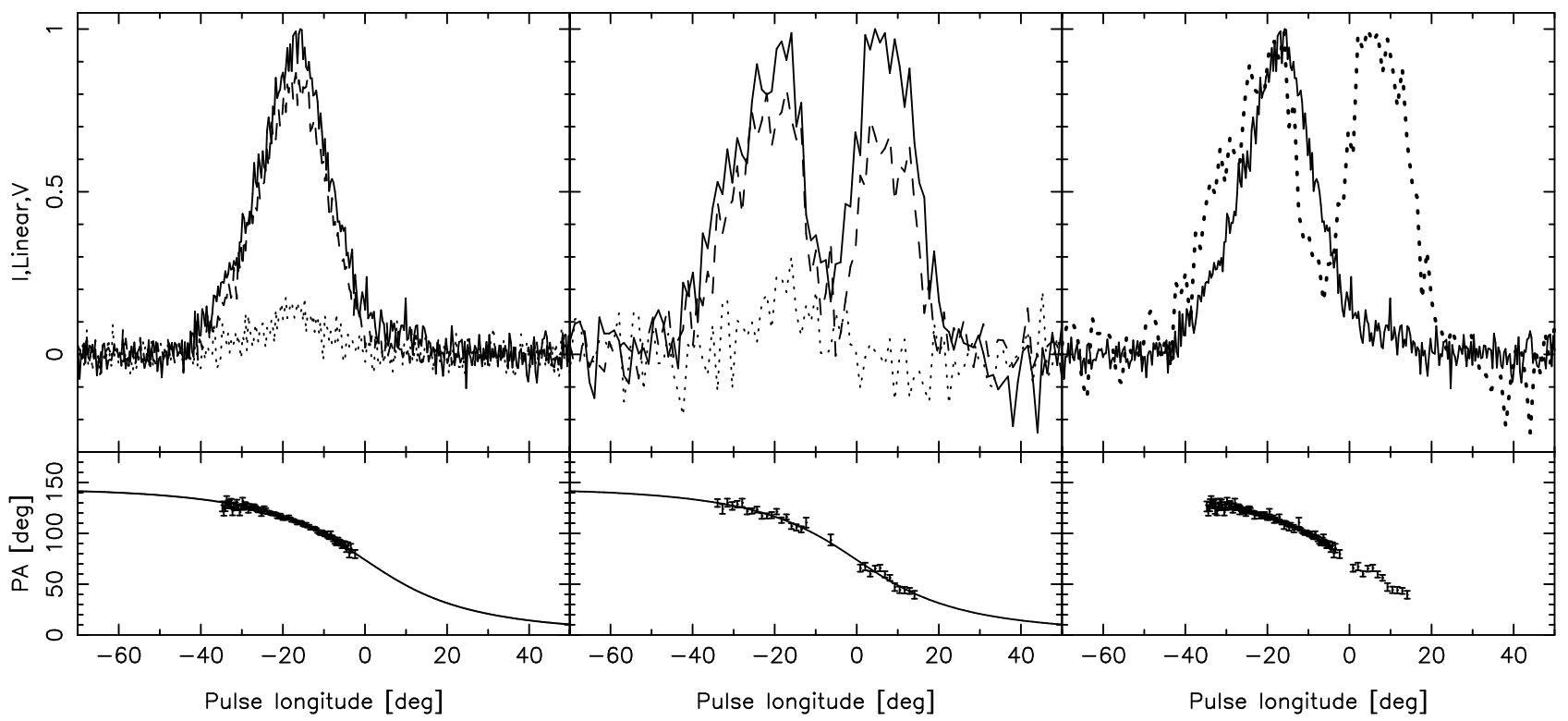

Figure 5. The high signal-to-noise standard profile showing the pulsar in its single-peaked mode (left panel) and the profile in its transient double-peaked mode (middle panel). The top plots show total intensity (solid line), linear polarization (dashed line) and circular polarization (dotted line). Zero longitude corresponds to the measured location of the steepest gradient of the PA curve (shown in the bottom panels). The bottom panel of the middle panel shows the RVM fit through the PA points. The exact same curve is shown in the left-hand panel, without any additional fitting applied. The perfect match, including the absence of any offset between the data and the model, unambiguously shows that it is the leading component of the double profile which corresponds to the single-peaked mode. Right panel: The total intensity profiles of the single-peaked mode (solid line) and the double-peaked mode (dotted line), as well as their PA points, are overlaid. The bottom panel illustrates again the perfect match of the PA-points.

showed a number of clearly detected bright individual pulses (bottom panel Fig. 4), while a 4 minute long 2009 August 9 observation does not show any sign of similar pulses.

The strong pulses appear to occur at three distinct pulse longitudes (approximately at $-30^{\circ},-10^{\circ}$ and $50^{\circ}$ ). Notice that the pulse profile of this particular observation shows structure at these pulse longitudes associated with the strong individual pulses (top panel of Fig. 4). This suggests that the strong pulses appear before and after the main peak of the observation. The relative alignment of this profile with respect to the single-peaked and double-peaked profile observed at a wavelength of $20 \mathrm{~cm}$ will be discussed in the next section in more detail. In total there are eight observations at a wavelength of $10 \mathrm{~cm}$ for which we have an integrated pulse profile and this observation is the only one which shows evidence for additional components in the pulse profile. The fact that the strong pulses do have corresponding peaks in the pulse profile suggests that at this frequency (or possibly epoch) the strong pulses are less RRAT-like than the strong pulses observed at a wavelength of $20 \mathrm{~cm}$.

Independent of whether single pulses occurred in one of the two, or eight, of the $10-\mathrm{cm}$ observations, given how rare the detection of individual pulses is at a wavelength of $20 \mathrm{~cm}$ and the fact that at $10 \mathrm{~cm}$ more individual pulses are detected suggests that strong pulses occur more often at higher frequencies. This is consistent with the fact that there are no individual pulses detected in two 3 minute observations made at a wavelength of $20 \mathrm{~cm}$ one day earlier and two days later. Only if the event rate is high for periods of time less than a day and we have been lucky during the observation at a wavelength of $10 \mathrm{~cm}$ the event rates could be the same at the two frequencies.

\section{POLARIZATION PROFILES}

It is not immediately obvious which of the two components of the double-peaked profile corresponds to the singlepeaked profile and therefore it is not clear if and how the newly emerged component relates to the RRAT-like emission. In principle one could use pulsar timing methods to resolve this issue, but, as will be discussed in Sect. 6] a large amplitude glitch makes this impossible. We therefore have to resort to other measurements to find out how to time align the normal single-peaked pulse profile and the doublepeaked profile. We will show in the this section that the position angle (PA) of the linear polarization can be used to find this relative alignment. Polarization data are further used to constrain the viewing geometry and the radio emission height.

\subsection{Alignment of the profile components}

Virtually all pulsars with $\dot{E}>2 \times 10^{35} \mathrm{erg} \mathrm{s}^{-1}$ have a linear polarization fraction over $50 \%$ (Weltevrede \& Johnston 2008; see also e.g. von Hoensbroech et al. 1998). In addition, most of these pulsars show a PA which varies smoothly as a function of pulse longitude forming a S-shape. This pulsar is no exception as one can see in the left panel of Fig. 5] The middle panel shows the double-peaked profile. Notice that the PA values of the pulsar in its normal mode 
clearly match those of the leading peak of the double-peaked profile, suggesting that the trailing peak is the component which has appeared in the double-peaked mode. Indeed a cross-correlation between the two profiles shows the highest degree of correlation for such an alignment. This is caused by the slight asymmetry seen for the profiles. In the singlepeaked mode the trailing side of the profile is slightly steeper caused by a weak emission component overlapping with the leading edge of the main peak (visible at $-35^{\circ}$ pulse longitude). The leading component in the double-peaked mode has also a shallower leading edge, although in that case there is no evidence for a weak component being responsible.

From the fact that the PA curve is very similar in the single- and double-peaked mode one can conclude that the PA provides a much stronger foundation to base the alignment of the two profiles on than the total intensity. In order to quantify the relative alignment of the two profiles we have fitted the data using the rotating vector model (RVM; Radhakrishnan \& Cooke 1969). In this model the PA of the linear polarization $\psi$ depends on the pulse longitude $\phi$ via

$$
\tan \left(\psi-\psi_{0}\right)=\frac{\sin \alpha \sin \left(\phi-\phi_{0}\right)}{\sin \zeta \cos \alpha-\cos \zeta \sin \alpha \cos \left(\phi-\phi_{0}\right)},
$$

where $\psi_{0}$ and $\phi_{0}$ are the PA and pulse longitude at the location where the $\psi(\phi)$ has its steepest gradient (and an inflection point). In addition, the shape of the PA curve depends on the emission geometry of the pulsar, which is described by two angles. Firstly the angle $\alpha$ between the magnetic axis and the rotation axis and secondly the angle $\zeta$ between the line-of-sight and the rotation axis. A related angle is the impact parameter $\beta=\zeta-\alpha$, which is the angle between the line-of-sight and the magnetic axis at its closest approach.

According to the RVM, $\phi_{0}$ corresponds to the pulse longitude at which the line-of-sight intersects the fiducial plane, the plane containing the rotation and magnetic axis. There is a longitude-shift one has to take into account if the emission height $h_{\mathrm{em}}$ is not negligible compared to the light cylinder distance, as will be discussed later. The longitude $\phi_{0}$ could act as a natural zero point for the pulse longitude scale if one uses the PA to align different profiles. This is achieved by determining $\phi_{0}$ by fitting Eq. 2 through the PA points. The profiles are then rotated such that the $\phi_{0}$ corresponds to $0^{\circ}$ in the plots of Fig. 5. Note that the alignment is independent of the actual value of the PA, it only depends on the pulse longitude corresponding with the steepest gradient of the RVM curve. This allows a self-consistency check of the determined alignment by over-plotting the RVM curve obtained from the fit of the double-peaked profile on the PA points of the single-peaked profile. One can see in Fig. 5 that this RVM curve matches both PA curves perfectly, something which is also evident from the right-hand panel which shows the PA points of the two profiles overplotted. This shows that the measured position of the steepest gradient of the PA curve is robust, because otherwise there would be a vertical (or horizontal) offset between the PA points of the two profiles.

Now that the single-peaked profile is aligned with the double-peaked profile, the leading RRAT-like component can be placed on the same longitude scale by aligning the main peaks. This is shown in Fig. [6 which shows both the sum of the four strong pulses observed at $20 \mathrm{~cm}$ (dotted line)

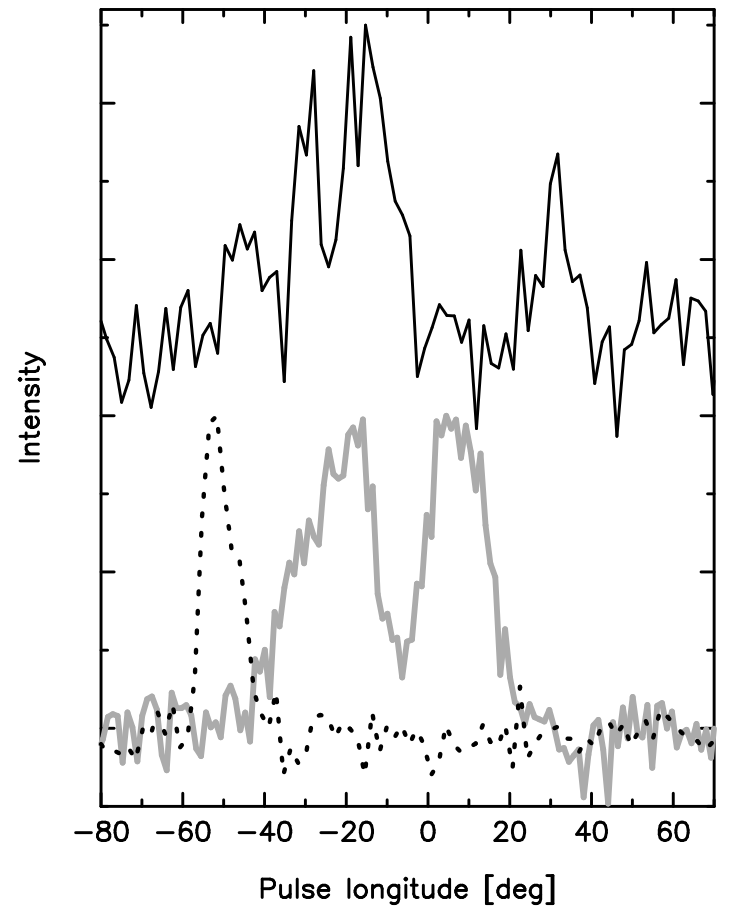

Figure 6. Plot showing the profiles obtained by averaging the four strong pulses shown in Fig.2(dotted line), the double-peaked profile (thick gray line) and the abnormal profile at $10 \mathrm{~cm}$ (solid line, with a vertical offset from the other two profiles). The pulse longitude scale is identical to that in Fig. 5 and the method for aligning the $10-\mathrm{cm}$ profile is explained in the text. The peak intensities of the profiles are normalized.

and the double-peaked profile (thick gray line) at the same wavelength. The strong pulses represent a distinct profile component with a pulse longitude separation from the main peak roughly equal to that between the main peak and the transient trailing peak of the double-peaked profile.

The remaining question is how the components seen in the $10-\mathrm{cm}$ observation discussed in Sect. 4.2 fit in this picture. The profile is far too weak to find the pulse longitude at which the gradient of the PA curve is steepest. Nevertheless, the value of the PA can be used to roughly align the profile to the PA curve observed at $20 \mathrm{~cm}$. It follows that the main peak of the $10-\mathrm{cm}$ profile overlaps roughly with the single-peaked profile observed at $20 \mathrm{~cm}$. In Fig. 6] the $10-\mathrm{cm}$ profile is shown together with the double-peaked profile and the RRAT-like component seen at $20 \mathrm{~cm}$. This alignment suggests that the bright pulses at the far leading edge of the $10-\mathrm{cm}$ profile correspond with the RRAT-like component seen at $20 \mathrm{~cm}$ (dotted line). The strong pulses seen at $10 \mathrm{~cm}$ at the far trailing edge of the profile appear to form another profile component, again with a similar component separation as between the other components. It should be emphasized that because of the low signal-to-noise ratio of the $10-\mathrm{cm}$ profile, its alignment with the other profiles should only be considered as a rough indication.

We therefore believe that although the pulse profile of PSR J1119-6127 is most of the time single-peaked, there are in fact at least four components. In addition to the normal emission, the profile has shown once a transient trailing 
Table 1. This table summarizes the considered choices for the location of the magnetic axis with respect to the pulse profiles of PSR J1119-6127. The second and third column denotes those longitudes in the single- and double-peaked mode respectively using the longitude shown used in Fig. 5. The next three columns indicate the implied emission height difference between the two modes and the emission heights themselves. The last column states which component of the double-peaked profile is the new component. In case II the single-peaked profile splits into two components. Both cases I and IV are argued to be plausible, case IV being our preferred solution.

\begin{tabular}{lccrcrl}
\hline \hline Case & $\phi_{\mathrm{sgl}}$ & $\phi_{\mathrm{dbl}}$ & $\begin{array}{c}\Delta h_{\mathrm{dbl}} \\
{[\mathrm{km}]}\end{array}$ & $\begin{array}{c}h_{\mathrm{sgl}} \\
{[\mathrm{km}]}\end{array}$ & $\begin{array}{r}h_{\mathrm{dbl}} \\
{[\mathrm{km}]}\end{array}$ & New comp. \\
\hline I & $-16^{\circ}$ & $-21^{\circ}$ & 400 & $1400^{a}$ & 1800 & Trailing \\
II & $-16^{\circ}$ & $-6^{\circ}$ & -900 & 1400 & 500 & Split \\
III & $-16^{\circ}$ & $+7^{\circ}$ & -2000 & 1400 & -600 & Leading \\
IV & $-6^{\circ}$ & $-6^{\circ}$ & 0 & 500 & 500 & Trailing \\
\hline
\end{tabular}

$a$ An emission height of $2200 \mathrm{~km}$ was derived by Johnston \& Weisberg (2006) with the steepest gradient lagging the peak of the total intensity profile by $26^{\circ}$. We attribute the discrepancy between their result and ours to the lower quality of their data and note that their pulse profile and PA curve are fully consistent with those presented in this paper. An emission height of $2100 \mathrm{~km}$ was derived by Weltevrede \& Johnston (2008), however in their table all emission heights derived from PA curves are overestimated by a factor 2 because of a miscalculation.

peak. These two peaks are flanked by two RRAT-like components which are usually inactive.

\subsection{Emission heights}

\subsubsection{0-cm emission}

The single-peaked profile does not perfectly match the leading component of the double-peaked profile as shown in the right-hand panel of Fig. 5 There is no a priori reason why they should match better, given that the shapes of the profiles are slightly different to start off with. On the other hand, there are reasons for the alignment of the profiles not to be perfect. If the emission height is different in the two states of the pulsar, which potentially could be the root-cause of the different profile shapes, one does expect a deviation in the alignment of the profiles. This is because only for a zero emission height $\phi_{0}$ corresponds to the pulse longitude at which the line of sight is passing the fiducial plane. For finite emission heights co-rotation of the emitting region makes the inflection point of the PA curve $\left(\phi_{0}\right)$ to be delayed with respect to the pulse profile. The relative shift $\Delta \phi$ between the pulse profile and the inflection point of the PA curve relates to the emission height $h_{\mathrm{em}}$ via (Blaskiewicz et al. 1991)

$$
h_{\mathrm{em}}=\frac{1}{4} R_{\mathrm{LC}} \Delta \phi=\frac{P c}{8 \pi} \Delta \phi .
$$

Here $P$ is the spin period of the pulsar, $c$ is the speed of light and $R_{\mathrm{LC}}$ is the light cylinder radius $(19,500 \mathrm{~km}$ for this pulsar). Therefore, if the emission heights are different in the single- and double-peaked modes, the pulse profiles will not be perfectly aligned when using the inflection point of the PA as the reference point.

In order for the centroids to overlap, the normal profile should be shifted by $5^{\circ}$ to earlier longitudes compared to the double-peaked profile. This corresponds to an emission height which is $400 \mathrm{~km}$ ( $2 \%$ of the light cylinder radius) lower in the single-peaked mode (case I in Table 1). Such an emission height difference would make both the PApoints and the total intensity profile overlap. If one allows the emission height difference between the two modes to be bigger, more extreme offsets could be considered. For instance, one could envision the fiducial plane to correspond to the centre of both the single-peaked and the double-peaked profile, thereby making both profiles symmetric around the magnetic axis (case II). Or even more extreme, maybe the single peak corresponds to the trailing component in the double-peaked mode (case III). In the final case we consider the magnetic axis corresponds to the symmetry point of the double-peaked profile without a differential emission height (case IV). With the made assumptions about which pulse longitude of the pulse profile corresponds to the fiducial plane, Eq. 3 can also be used to constrain the emission height itself rather than just height differences. The resulting emission heights of the single- and double-peaked mode can be found in Table 1 .

Given the fact that the component width is not very different in the normal mode and the double-peaked mode, one could argue that the emission height difference between the two modes must be relatively small compared to the total emission height. For case II (i.e. the single-peaked profile splits in the double-peaked mode, arguably the most symmetric scenario) one would expect the overall pulse width to be roughly 1.7 times larger in the single-peaked mode because of the curvature of the dipolar magnetic field lines. This effect must be very precisely counter-balanced by having a smaller fraction of the polar cap being active during the single-peaked mode in order to have similar component widths, at which point the model becomes contrived. This problem becomes even worse in case III, which is already unlikely given the negative derived emission height in the double-peaked mode.

In case IV a differential emission height cannot explain the apparent small offset between the single-peaked profile and the leading component of the double-peaked profile, as an essentially zero emission height would be required for the single-peaked mode. Nevertheless, the striking mirror symmetry of the double-peaked profile can be seen as strong evidence for the location of the fiducial plane at that pulse longitude.

Therefore we conclude that, even when differential emission heights are considered, the most likely scenario is that it is the trailing peak which has emerged in the double-peaked mode (either case I or IV in Table 1). Possibly (although not necessarily) the fiducial plane corresponds to the exact centroid of both the profile of the single-peaked mode and the leading component in the double-peaked mode (case I). Otherwise the emission height might be identical in the two modes with the fiducial plane corresponding to the centre of the double-peaked profile (case IV).

\subsubsection{0-cm emission}

Fig. 7 shows the standard profiles obtained from the timing program at a wavelength of 20 and $10 \mathrm{~cm}$. The longitude scale used for the $20-\mathrm{cm}$ profile is the same as in Fig. 5 and the alignment of the $10-\mathrm{cm}$ profile is obtained by matching 


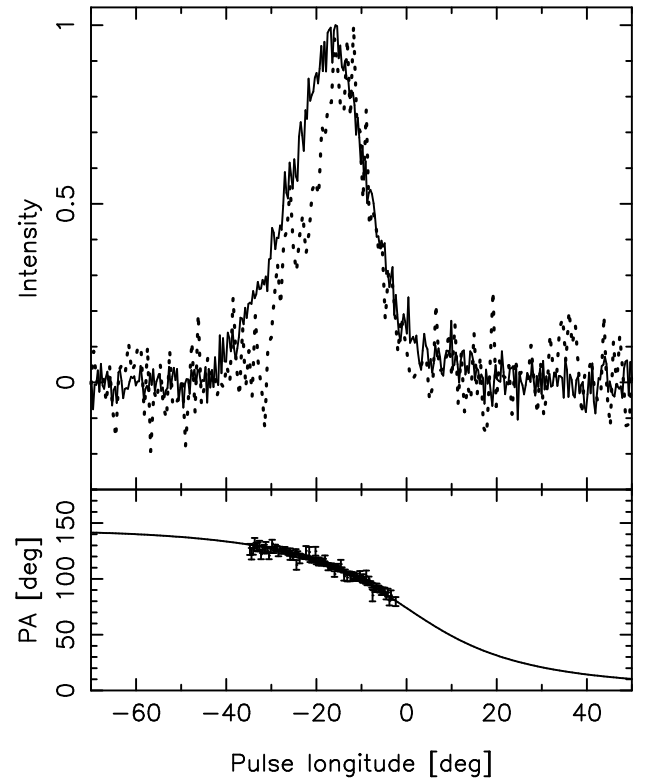

Figure 7. The normal profile of PSR J1119-6127 at a wavelength of $20 \mathrm{~cm}$ (solid line) and at $10 \mathrm{~cm}$ (dotted line). The steepest gradient of the PA-swing measured at a wavelength of $20 \mathrm{~cm}$ occurs at $0^{\circ}$ longitude. The PA-points of the $10 \mathrm{~cm}$ data were aligned by eye to those at $20 \mathrm{~cm}$ and the PA-points at both frequencies are overlaid in the bottom panel. The alignment of the profiles at the two frequencies is, within the uncertainties given the relatively low signal-to-noise ratios, the same as in Fig. 6

the PA curves of the $10-$ and $20-\mathrm{cm}$ profiles. The centroid of the $10-\mathrm{cm}$ profile appears to be shifted to later longitudes compared to the $20-\mathrm{cm}$ profile by about $3^{\circ}$. Using the same methodology as described earlier, this suggests that the emission height is $300 \mathrm{~km}$ lower at a wavelength of 10 $\mathrm{cm}$. This height difference implies a difference in the expected half opening angle $\rho$ of the beam at the two wavelengths when using the equation

$$
\rho=\sqrt{\frac{9 \pi h_{\mathrm{em}}}{2 P c}} .
$$

In this equation $\rho$ is related to the opening angle of the last open dipole field lines at the emission height (e.g. Lorimer \& Kramer 2005), thereby assuming that the full polar cap is active.

The difference in emission height implies that the emission height at a wavelength of $20 \mathrm{~cm}$ is a factor of 1.2 and 2.0 higher than that of the $10-\mathrm{cm}$ emission for cases I and IV of Table 1 respectively, which translates in a factor 1.1 and 1.4 difference in $\rho$. The latter factor is similar to the observed difference in pulse width at the two wavelengths, therefore supporting scenario IV unless the line of sight is grazing the edge of the emission cone. It should be stressed that this is not a very direct argument, as it involves a chain of assumptions such as the assumption that the same field lines are producing radio emission at the two frequencies. Nevertheless, the data are at least consistent with case IV.

\subsection{Viewing geometry}

The orientation of the radio beam with respect to the line of sight and the rotation axis can be quantified with the

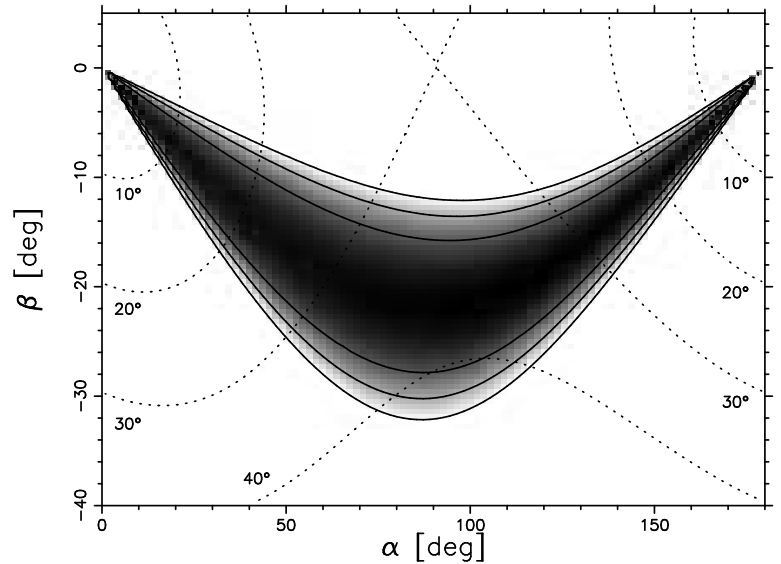

Figure 8. The $\chi^{2}$ map resulting from fitting the RVM model to the polarization data is shown in grayscale. The solid contours indicate where the $\chi^{2}$ is two, three and four times larger than the minimum value. Overlaid are the $\rho$ contours assuming the double-peaked pulse profile fills the last open field line region.

angles $\alpha$ and $\zeta$. These angles can be constrained first of all by fitting the RVM curve (Eq. 2). This is visualized in Fig. 8 which shows the $\chi^{2}$ of the RVM fit. As is often the case, the contours are banana-shaped, caused by a lack in pulse longitude coverage of the PA points. Therefore, not surprisingly, the best constraint is obtained by using the PA curve of the double-peaked profile rather than the singlepeaked standard profile despite the much higher signal-tonoise ratio of the latter.

An additional constraint follows from estimated half opening angle $\rho$ of the beam (Eq. 4), which constrains the angles $\alpha$ and $\zeta$ because $\rho$ is related to the pulse width $W$ via

$$
\cos \rho=\cos \alpha \cos \zeta+\sin \alpha \sin \zeta \cos (W / 2),
$$

assuming the radio beam is symmetric about the magnetic axis (Gil et al. 1984; Lorimer \& Kramer 2005). The dotted contours in Fig. 8 indicate the $\rho$ values as derived using Eq. 5. using the observed pulse width of the double-peaked profile (which we take to be the full longitude range over which we observe significant emission).

In case I the radio beam is asymmetric in the doublepeaked mode (but symmetric in the single-peaked mode). Taking the outer edge of the trailing component of the double-peaked profile to correspond to the last open field line and using $\rho=26^{\circ}$ (the expected opening angle for an emission height of $1800 \mathrm{~km}$ in the double-peaked mode) it is derived that $\alpha \sim 30^{\circ}-40^{\circ}$. Note that this implies that the strong pulses at the trailing edge of the profile observed at $10 \mathrm{~cm}$ are produced outside the polar cap region. If all emission occurs from within the open field line region the effective pulse width is larger making $\alpha$ smaller.

For case IV in Table 1, the solution we preferred in subsection 5.2 $\rho=14^{\circ}$ (Eq. 4). This suggests $\alpha=20^{\circ}-30^{\circ}$ (see Fig. 8) assuming the double-peaked profile fills the open field line region. If the RRAT-like emission comes from open field lines a more aligned geometry $\left(\alpha \simeq 17^{\circ}\right)$ is required to explain the larger overall pulse width (see Fig. 6). 


\section{TIMING}

In order to characterise the spin-behaviour of a pulsar one needs to measure the time-of-arrival (TOA) of the radio pulses as a function of time. This is done by cross correlating the pulse profiles of the individual observations with a high signal-to-noise radio standard profile (shown in the left panel of Fig. 5). Clock corrections were applied to the TOAs which were then converted to arrival times at the solar system barycentre using the DE405 model (Edwards et al. 2006; Standish 1998) using the TEMPO2 timing package (Hobbs et al. 2006).

Next, the barycentric TOAs are compared with a timing model and its parameters are refined by minimising the socalled timing residuals. The refining process has been done using custom software which in essence works identical to timing packages such as TEMPO2. The minimisation procedure makes use of the downhill simplex method and the uncertainties are derived from the correlation matrix which is estimated by calculating the Hessian matrix (e.g. Press et al. 1992).

The most basic timing model for the rotation of a pulsar is a truncated Taylor series:

$$
\phi(t)=\phi_{0}+\nu\left(t-t_{0}\right)+\frac{1}{2} \dot{\nu}\left(t-t_{0}\right)^{2}+\frac{1}{6} \ddot{\nu}\left(t-t_{0}\right)^{3},
$$

where $\phi(t)$ is the rotational phase as function of time. The parameters $\phi_{0}, \nu, \dot{\nu}$ and $\ddot{\nu}$ are the rotational phase, spin frequency and its first two time derivatives, all defined at epoch $t_{0}$, usually taken to be during the time covered by the observations.

PSR J1119-6127 presents a typical timing behaviour of a pulsar of its age in the sense that like the Crab and other very young pulsars it exhibits a relatively stable spindown $(|\dot{\nu}(t)|)$ evolution despite the significant timing noise observed. This allows a good estimate of its braking index as will be discussed later. During the time of the observations the rotation of PSR J1119-6127 has been interrupted by two major glitches exhibiting a rather particular behaviour.

A pulsar glitch is a sudden, normally unresolved, increase of the rotation frequency (Radhakrishnan \& Manchester 1969). Sometimes, glitches are observed to be followed by an exponential-like recovery towards the former rotational state; phenomenon that has been interpreted as a signature of the presence of a superfluid in the interior of the star (Baym et al. 1969). In many cases this recovery is either too small or the sampling too sparse to quantify its effects.

The two large glitches that we report here occurred in 2004 and 2007; the first one between MJD 53279 and 53306 (October 1 and 28), and the second one in 2007 between MJD 54220 and 54268 (April 30 and June 17). All TOAs between MJD 50850 and 55364 were modelled using a single model that includes these glitches. The phase difference after a glitch is modelled using an additional phase $\phi_{\mathrm{g}}(t)$, which describes the deviation from Eq. 6 caused by the glitch. The phase after the event is parametrised by permanent jumps in frequency $\left(\Delta \nu_{p}\right)$ and frequency derivative $\left(\Delta \dot{\nu}_{p}\right)$ plus an exponential decay with time constant $\tau_{d}$ :

$$
\begin{aligned}
\phi_{\mathrm{g}}(t)= & \Delta \phi+\Delta \nu_{p}\left(t-t_{g}\right)+\frac{1}{2} \Delta \dot{\nu}_{p}\left(t-t_{g}\right)^{2} \\
& -\Delta \nu_{d} \tau_{d} e^{-\left(t-t_{g}\right) / \tau_{d}}
\end{aligned}
$$

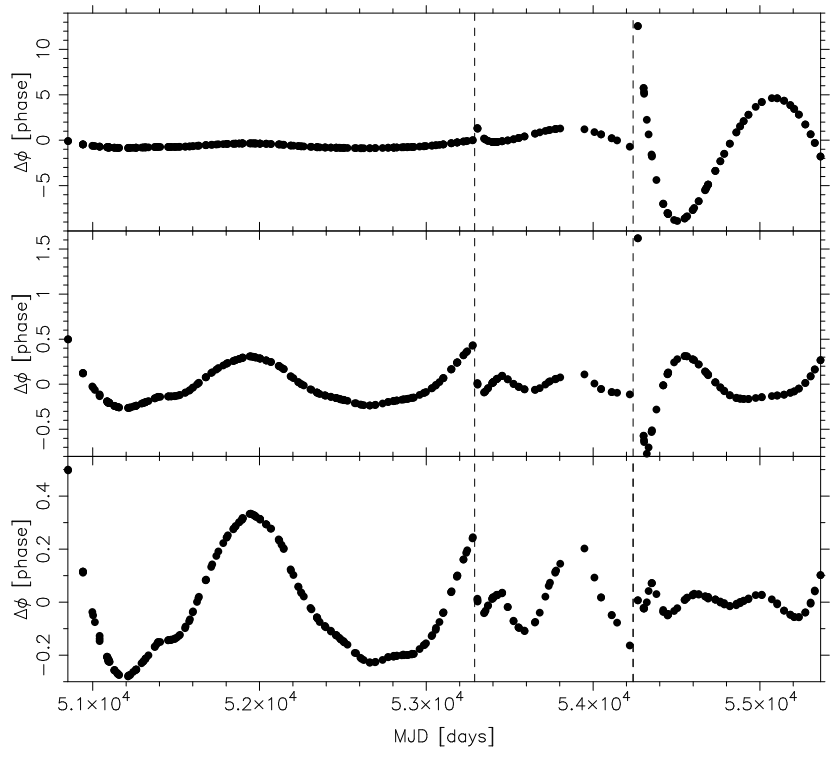

Figure 9. Top panel: The timing residuals obtained by only modelling the permanent jumps in rotational frequency and its time derivative after the two glitches (their epoch is indicated by the dashed lines). The ordinate is expressed in turns. Middle panel: Timing residuals obtained by including a single exponential glitch recovery after each glitch. Bottom panel: Timing residuals obtained by including a glitch recovery on a second faster timescale after the second glitch.

where $t_{g}$ corresponds to the glitch epoch and $\Delta \nu_{d}$ is the step in frequency which is exponentially recovered. The step in rotational phase $\Delta \phi$ is not a physical jump in phase, but it corrects for a $t_{g}$ which is generally not specified accurate to a fraction of a rotational period. The instantaneous frequency jump at the glitch epoch is given by $\Delta \nu_{g}=\Delta \nu_{p}+\Delta \nu_{d}$ and the corresponding frequency derivative jump is $\Delta \dot{\nu}_{g}=$ $\Delta \dot{\nu}_{p}+\Delta \dot{\nu}_{d}$, where $\Delta \dot{\nu}_{d}=-\Delta \nu_{d} / \tau_{d}$.

During the minimisation process the epoch of the two glitches were kept fixed at the values MJD 53290 and 54240, respectively. The top panel of Fig. 9 shows the timing residuals after applying the above described model having set $\Delta \nu_{d}=0$, i.e. neglecting any possible glitch recovery. It is clear that the amplitude of the residuals is much larger after both glitches, indicating that the glitches are poorly modelled. This, in combination with the observed pattern of the residuals, is suggestive of a post-glitch transient stage. Accordingly, the residuals are considerably improved when exponential recoveries are allowed for the two glitches (middle panel of Fig. 91). Nevertheless, the residuals immediately after the 2007 glitch show significant deviations, indicating that the recovery still has not been properly modelled. If a second exponential recovery term with a shorter time constant is included for this glitch, the residuals become much smaller (bottom panel of Fig. 91). The parameters of this last fit can be found in Table 2 Because the recovery process may not yet be complete at the time of writing, the parameters for the 2007 glitch may change slightly if new data are added in the near future.

As mentioned earlier, the timing analysis can in principle be used to identify which component of the doublepeaked profile corresponds to the normal profile. This can 

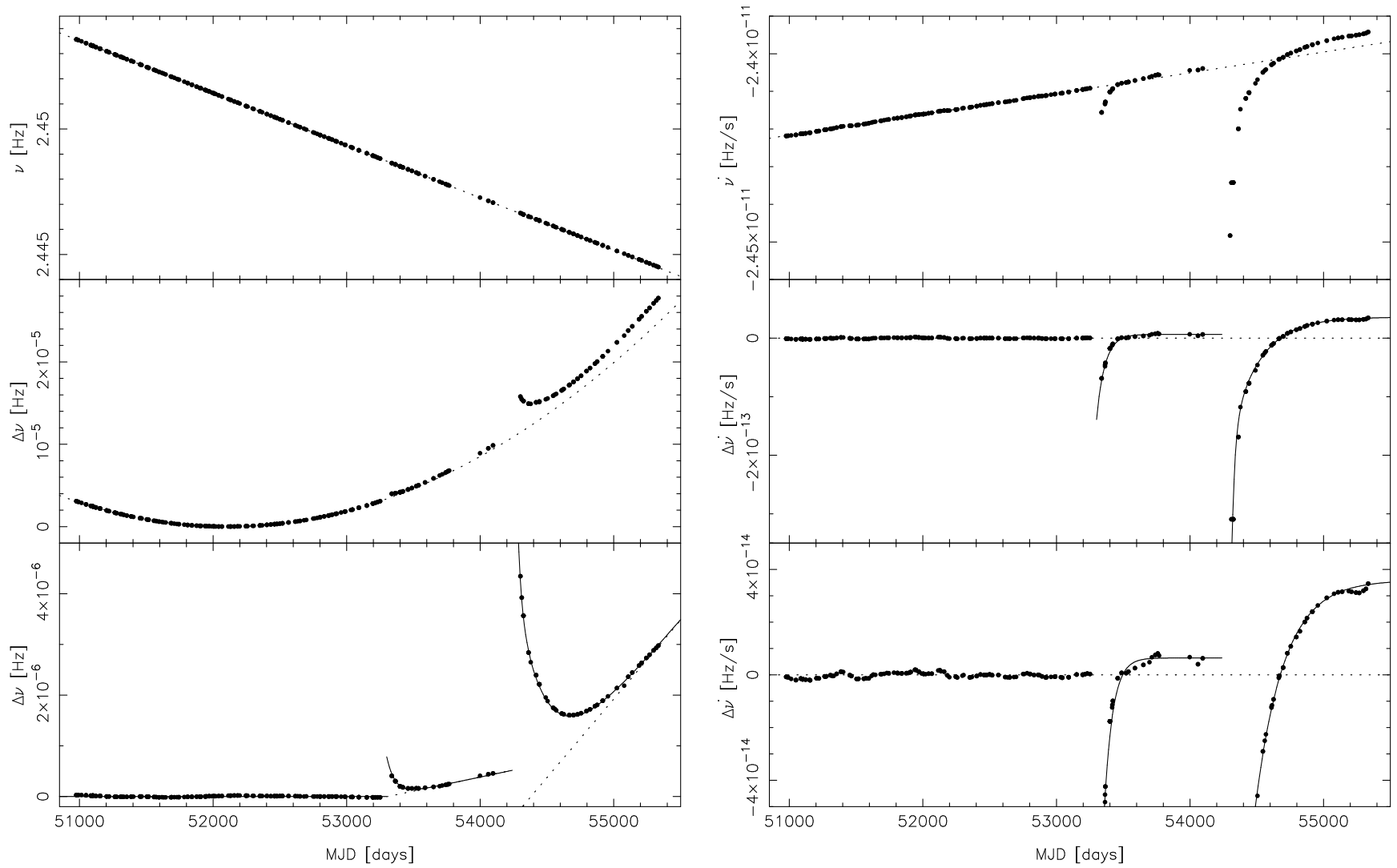

Figure 10. Top-left panel: The measured rotational frequency of PSR J1119-6127 as a function of time. The frequency is decreasing, as is expected from spin-down. The prediction according to the measured $\nu$ and $\dot{\nu}$ in Table 2 is shown as a dotted line. Middle-left panel: The effect of the constant slow-down is subtracted from the rotational frequency. Especially the second glitch can clearly be seen as a deviation from the parabola-shape. The latter indicates a significant and stable braking-index and the prediction according to the measured $\ddot{\nu}$ is shown as a dotted line. Bottom-left panel: The difference between the measured spin frequency and the contributions from $\nu, \dot{\nu}$ and $\ddot{\nu}$. The solid and dotted lines show the prediction according to the glitch model including/excluding glitch recovery. Top-right panel: The measured spin-frequency derivative as a function of time. The dotted line indicates the $\dot{\nu}$ and $\ddot{\nu}$ contribution. Middle-right panel: The difference between the data and the dotted line of the top-right panel. The solid line indicates the prediction according to the glitch model including glitch recovery. Bottom-right panel: This plot is identical to the middle-right panel, but slightly zoomed in. It can clearly be seen that the exponential glitch-recovery is over relaxing resulting in a positive step in spin-frequency derivative, i.e. the pulsar spins down slower than what could be expected from its braking index.

be done by calculating a TOA for both peaks and by determining which corresponding residual follows the general trend of the residuals closest. However, the double-peaked profile was detected in the first observation after the 2007 glitch, hence during the fast part of the recovery process. This makes it impossible to distinguish between the residuals corresponding to the two peaks because both residuals can be explained equally well by slightly adjusting the glitch parameters. The timing solution presented in Table 2 therefore makes use of the alignment discussed in Sect. 5.1

\subsection{Glitch properties}

Fig. 10 shows the rotational evolution of PSR J1119-6127 over more than 12 years. Its spin parameters as a function of time were measured using short stretches of data. For each TOA we took all TOAs within a range of 75 days and minimized the residuals using a timing model that only included $\phi_{0}, \nu$ and $\dot{\nu}$ as free parameters and $t_{0}$ as a fixed parameter. Fig. 10] only includes points which are based on more than three TOAs.
The top left panel of Fig. 10 shows the frequency against time, which decreases steadily according to the measured $\dot{\nu}$. However, the spin-down rate varies slightly with time and the effects of $\ddot{\nu}$ are only visible when subtracting the effects of $\dot{\nu}$ first, as shown in the middle-left panel. The almost perfect parabola-shape implies a rather constant and stable second spin-frequency derivative, from which the braking index can be estimated. In this panel it is also possible to see the effects of the two large glitches mentioned above. Both glitches exhibit recoveries towards the pre-glitch trend, as commonly observed in many young pulsar glitches (Shemar \& Lyne 1996). However, it can be observed that especially after the 2007 glitch the rotation evolves towards a new state, involving a lower spin-down rate $|\dot{\nu}|$ and therefore showing a larger spin-frequency than the pulsar would have if it continued evolving as before the glitch (see the bottom-left panel). This particular behaviour is best appreciated by looking at the evolution of $\dot{\nu}$, as shown in the top-right panel. The spin-down rate evolves almost linearly, as expected for a stable $\ddot{\nu}$, only interrupted by the two major glitches and their recoveries. After each glitch there is a 
Table 2. Rotational parameters for PSR J1119-6127.

\begin{tabular}{lc}
\hline \hline Parameter & Value \\
\hline Epoch (MJD) & 52109.85 \\
$\nu(\mathrm{Hz})$ & $2.4512027814(5)$ \\
$\dot{\nu}\left(10^{-15} \mathrm{~Hz} \mathrm{~s}^{-1}\right)$ & $-24155.07(1)$ \\
$\ddot{\nu}\left(10^{-24} \mathrm{~Hz} \mathrm{~s}^{-2}\right)$ & $638.9(4)$ \\
$\phi_{0}$ & $37.61(2)$ \\
$\mathrm{DM}\left(\mathrm{cm}^{-3} \mathrm{pc}\right)$ & 713 \\
$n$ & $2.684(2)$ \\
MJD range & $50850-55364$ \\
\hline 2004 glitch parameters & \\
\hline Glitch epoch & 53290 \\
$\Delta \phi$ & $4.8(3)$ \\
$\Delta \nu_{p}(\mu \mathrm{Hz})$ & $-0.005(13)$ \\
$\Delta \dot{\nu}_{p}\left(10^{-15} \mathrm{~Hz} \mathrm{~s}^{-1}\right)$ & $6.4(3)$ \\
$\Delta \nu_{d}(\mu \mathrm{Hz})$ & $0.8(1)$ \\
$\tau_{d}($ days $)$ & $63(8)$ \\
\hline 2007 glitch parameters & \\
\hline $\mathrm{Glitch} \mathrm{epoch}$ & 54240 \\
$\Delta \phi$ & $22(3)$ \\
$\Delta \nu_{p}(\mu \mathrm{Hz})$ & $-1.0(1)$ \\
$\Delta \dot{\nu}_{p}\left(10^{-15} \mathrm{~Hz} \mathrm{~s}^{-1}\right)$ & $30(1)$ \\
$\Delta \nu_{d}^{(1)}(\mu \mathrm{Hz})$ & $5.1(1)$ \\
$\tau_{d}^{(1)}($ days $)$ & $214(7)$ \\
$\Delta \nu_{d}^{(2)}(\mu \mathrm{Hz})$ & $9(2)$ \\
$\tau_{d}^{(2)}($ days $)$ & $23(3)$ \\
\hline
\end{tabular}

jump in spin-down rate followed by an exponential recovery to pre-glitch values. However, as indicated by the positive permanent jump $\Delta \dot{\nu}_{p}$ (Table 2), after the recovery of the 2007 glitch the spin-down rate is smaller than the projected pre-glitch value by almost $30 \pm 1 \times 10^{-15} \mathrm{~Hz} \mathrm{~s}^{-1}$ (see the last two panels at the right hand side of Fig. 10). The recovery of the 2004 glitch also presents this behaviour, as indicated by its positive $\Delta \dot{\nu}_{p}=6.4 \pm 0.3 \times 10^{-15} \mathrm{~Hz} \mathrm{~s}^{-1}$.

This post-glitch behaviour is not normal among the rest of the pulsar population and it has been clearly observed only for the RRAT PSR J1819-1458 (Lyne et al. 2009). Normally, if a glitch recovery is detected, $\Delta \dot{\nu}_{p} \leqslant 0$, i.e. the spindown rate either goes back to values similar to those before the glitch or stays at higher rates for a longer time (see Fig. 6 in Lyne et al. 2009).

The parameter $Q=\Delta \nu_{d} / \Delta \nu_{g}$ is used to quantify the degree of recovery of the spin frequency (e.g. Shemar \& Lvne 1996) and it is observed that pulsars exhibit $Q<1$ for most glitches (e.g. Lyne et al. 2000). Because some of the glitch parameters depend on the glitch epoch, the uncertainty on $Q$ is estimated by obtaining another 4 solutions (in addition to the one presented in Table 2) by varying the glitch epochs in the range defined by the TOAs just before and after each glitch. The $\mathrm{Q}$ parameters obtained are both greater than one: $Q=1.01 \pm 0.01$ for the 2004 event and $Q=1.1 \pm 0.1$ for the 2007 one. These anomalous values are due to the negative permanent frequency jumps $\Delta \nu_{p}$ observed for both glitches.

A new parameter $Q^{\prime}=\Delta \dot{\nu}_{d} / \Delta \dot{\nu}_{g}$ can be introduced to quantify the recovery of the spin-down rate. By making use of the relation $\Delta \dot{\nu}_{d}=-\Delta \nu_{d} / \tau_{d}$, it can be re-written as

$$
Q^{\prime}=\frac{\Delta \nu_{d}}{\Delta \nu_{d}-\tau_{d} \Delta \dot{\nu}_{p}} .
$$

Because $\Delta \dot{\nu}_{p}$ is usually a negative quantity, normal glitches satisfy $Q^{\prime}<1$. The $Q^{\prime}$ values of the 2004 and 2007 glitches are $1.046 \pm 0.004$ and $1.01 \pm 0.02$ respectively. As with $Q$, uncertainties are derived by using the 5 different timing solutions (see above). It is worth noticing that if $\Delta \dot{\nu}_{p}>0$, as is the case for these two glitches, then $Q^{\prime}$ will be always greater than unity. The amount of overpassing reflects the relative size of the recovered jump $\Delta \dot{\nu}_{d}$ respect to the instantaneous jump $\Delta \dot{\nu}_{g}$. Consequently, even though the anomalous recovery is more dramatic for the 2007 glitch (large $\Delta \dot{\nu}_{p}$ ), $Q^{\prime}$ is larger for the 2004 glitch.

We note that both $Q$ and $Q^{\prime}$ are strongly dependant upon the exponential fit, including the glitch epoch. Changes in the model or the addition of more post-glitch data could therefore modify their values considerably. Nonetheless, the fact they are greater than unity is significant (despite their relatively large uncertainties), since it depends purely upon the sign of the permanent jumps $\Delta \nu_{p}$ or $\Delta \dot{\nu}_{p}$ which are measured with much higher confidence. In other words, the anomalous behaviour of these two glitches is already well described by the signs of the permanent jumps.

We note that Camilo et al. (2000) report a small glitch that occurred in 1999 at MJD 51400. This glitch is much smaller $\left(\Delta \nu_{g}=0.011 \pm 0.001 \mu \mathrm{Hz}\right)$ than the glitches we report here. Although there is clearly a timing deviation at that epoch, Figs. 9 and 10 do not show convincing evidence that this glitch is significantly different from other timing irregularities. Indeed, there was no need to include this glitch in the long term timing solution (Table 2).

\subsection{Long-term spin evolution}

The long term spin evolution of pulsars can be characterised by the so-called braking index $n$, defined by the power law $\dot{\nu}=-k \nu^{n}$, where $k$ is a positive constant. With this definition the braking index can be estimated from the observable rotational frequency and its first two derivatives, since differentiation of the power law gives $n=\nu \ddot{\nu} / \dot{\nu}^{2}$. Different braking mechanisms will produce different values of $n$ (Blandford \& Romani 1988), and it is found that most measured braking indices are less than 3 , the canonical value for pure electromagnetic torque (e.g. Livingstone et al. 2007).

As it happens with most known very young pulsars, the variation of the spin-down rate $(\ddot{\nu})$ is significant and stable, and therefore relatively easy to measure. Camilo et al. (2000) measured this variation for PSR J1119-6127 and reported a braking index $n=2.91 \pm 0.05$. Using the timing solution presented in Table 2 we obtain a braking index $n=2.684 \pm 0.002$, the error calculated by propagating the individual errors on the frequency and its derivatives. While the value reported by Camilo et al. (2000) was measured using a data span of about $1.2 \mathrm{yr}$, this new estimate represents the spin behaviour of the pulsar for more than 12 years, hence the improvement of the uncertainty. The two large glitches interrupted the spin-down evolution carried on during the first half of the data (see Fig 10), and it is not obvious how they will affect the long term spin evolution. If only data until the first large glitch is used, the result is $n=2.686 \pm 0.002$, well contained between the error margins of the value given above. This similarity makes us confident that the glitches are modeled in a physically sensible way.

We measure a braking index $n=2.684 \pm 0.002$, smaller than the predicted value for pure dipolar electromagnetic 
braking, which implies the presence of extra torques. The long-term spin evolution of this pulsar is however unclear, and may not be solely dictated by the measured braking index. If glitches like the one reported here are normal, the effective long-term evolution would have to be described by a larger braking index. In that case, the quoted value would better represent the inter-glitch, rather than the long-term spin evolution.

\section{DISCUSSION}

\subsection{The glitch-induced identity changes of PSR J1119-6127}

One of the most intriguing properties of PSR J1119-6127 is that the radio emission of its distinct profile components can be classified differently. Its profile is most of the time single peaked and in no way remarkable. Only once in more than 12 years of data a very different pulse profile was observed with a second transient peak appearing next to the "normal" leading peak. The switching timescale is therefore more similar to those of the intermittent pulsars than the shorter timescale mode changes observed in many other pulsars. The leading component in the transient double-peaked mode is usually active at radio wavelengths and it is the trailing peak which has emerged. This identification is made by comparing the PA curve in the normal and the doublepeaked mode. In addition, the circular polarization and the profile shape show a similar correlation.

Radio emission which can be put into a third category was discovered by analysing the individual pulses of this source. In a single observation made at a wavelength of 20 cm four strong pulses leading both the "normal" and "intermittent" component were detected. These pulses are sporadic enough to make the signal disappear in the noise when adding the emission of all rotations during the observation together, thereby making this a "RRAT-like" component. At a wavelength of $10 \mathrm{~cm}$ a second component showing extremely erratic emission was detected trailing the other profile components, thereby making these erratic components flank the other emission.

The "identity changes" of PSR J1119-6127 are clearly glitch-induced. Not only it has been demonstrated that the rate of occurrence of the RRAT-like pulses at a wavelength of $20 \mathrm{~cm}$ must have been significantly higher around the epoch of their detection, but more importantly, all abnormal behaviour is observed directly after the large amplitude glitch. The transient component was detected in 2007 June 17 (once in 49.8 hours of data), which is the first observation after the glitch. The leading RRAT-like component was detected at a wavelength of $20 \mathrm{~cm}$ in 2007 August 20 (only four pulses out of the 246,892 inspected stellar rotations in total). This observation was the first long individual pulse recording after the glitch at a wavelength of $20 \mathrm{~cm}$ (3211 pulses long). Three other $20 \mathrm{~cm}$ observations were made between this observation and the glitch epoch. However, with a total of only 945 recorded stellar rotations and the relatively low event rate it is not surprizing the RRAT-like pulses were only detected during the longer observation. Finally, the trailing and leading erratic components were detected in 2007 July 23 at a wavelength of $10 \mathrm{~cm}$, which is the first $10 \mathrm{~cm}$ observation after the glitch. This makes it likely that erratic RRAT-like pulses were emitted throughout the time between the glitch epoch and their detection at $20 \mathrm{~cm}$.

Because of the separation between the observing sessions, the glitch epoch cannot be accurately determined. As far as we can tell, the abnormal emission properties of PSR J1119-6127 started directly after the glitch and continued for $78 \pm 24$ days. This corresponds to $4 \pm 1$ times the timescale of the fast part of the recovery or $0.4 \pm 0.1$ times the timescale of the slow part of the recovery. This is the first time that a glitch, or the post-glitch recovery process, is observed to influence the radio emission process of a normal (non-RRAT) pulsar.

It is currently not possible to know for sure how different the outer erratic components are compared to the intermittent second peak. Both phenomenon are observed around the same epoch and appear to be transient events and could therefore represent different phases of the same phenomenon. The additional components probably represent regions on the polar cap which usually fail in producing the positron-electron pairs required for the emission mechanism to work. One could speculate that a large-amplitude glitch for some reason changes the magnetosphere or stellar surface such that these regions switch-on, either completely or in a flickering manner. Possibly, for all large glitches the steady emission is followed by a flickering state, before the physical conditions relax back to the pre-glitch state and the components switch off again. It must be noted that the overall profile symmetry with the more steady components in the centre and the more flickering components at the edges of the beam suggests that the different components do have distinct emission properties rather than we observing the different components in different phases of the same cycle.

It is not clear why the occurrence of a glitch and the changes in the radio emission are related to each other. If the glitch is triggered solely by the conditions inside the star, than how is the energy involved in the glitch transported to the magnetosphere to induce the changes in the emission properties? This problem could suggest that there is an external event (a change in the magnetosphere) that triggers both the glitch and the changes in the emission properties. In the next subsection we will argue that the glitches might be triggered by magnetic stresses on the crust.

\subsection{The glitches of PSR J1119-6127}

\subsubsection{Comparison with other pulsars}

Detected glitch frequency jumps $\Delta \nu_{g}$ are in the range $0.001-$ $50 \mu \mathrm{Hz}$ among the pulsar population, implying fractional changes $\Delta \nu_{q} / \nu$ between $10^{-11}$ and $10^{-5}$ (Shemar \& Lyne 1996; Wang et al. 2000). Young pulsars like the Crab and PSR J1119-6127 normally exhibit smaller glitches, with $\Delta \nu / \nu<300 \times 10^{-9}$. However, the 2007 glitch, with $\Delta \nu_{g} / \nu \sim$ $5400 \times 10^{-9}$, is as big as the largest glitches detected in the rotation of the Vela pulsar and many other young pulsars with characteristic ages between 10 and $100 \mathrm{kyr}$.

Exponential glitch recoveries have been observed before for other pulsars and some of them, like PSR J11196127, have required more than one time constant. For instance, more than one timescale was necessary to model the very large glitch in PSR B2334+61 (Yuan et al. 2010) 
Table 3. Recovery parameters for PSR J1119-6127 and other pulsars. Uncertainties for the last quoted digit are given in parenthesis. Note that the fact that $Q$ and $Q^{\prime}$ of PSR J1119-6127 are larger than one is significant, despite the large error bars on the values themselves (see text).

\begin{tabular}{|c|c|c|c|c|c|c|c|}
\hline PSR & $\begin{array}{l}\text { MJD } \\
{[\text { days] }}\end{array}$ & $\begin{array}{l}\Delta \nu_{g} / \nu \\
\left(10^{-6}\right)\end{array}$ & $\begin{array}{c}\Delta \nu_{p} \\
{[\mu \mathrm{Hz}]}\end{array}$ & $\begin{array}{c}\Delta \dot{\nu}_{p} \\
{\left[10^{-15} \mathrm{~Hz} \mathrm{~s}^{-1}\right]}\end{array}$ & $Q$ & $\overline{Q^{\prime}}$ & Refs. \\
\hline J1119-6127 & 54240 & $5.4(8)$ & $-1.0(1)$ & $30(1)$ & $1.1(1)$ & $1.01(2)$ & - \\
\hline J1819-1458 & 53924.8 & $0.699(4)$ & $0.138(1)$ & $0.79(1)$ & $0.159(4)$ & $1.7(5)$ & 1 \\
\hline J1846-0258 & 53883 & $4(1)$ & $-95(1)$ & $-274(13)$ & $9(3)$ & $0.973(2)$ & 2,3 \\
\hline B0355+54 & 46497 & $4.368(2)$ & $27.8773(1)$ & $-0.5(1)$ & $0.00117(4)$ & $0.14(3)$ & 4 \\
\hline B1800-21 & 48245 & $4.07(2)$ & $30.07(1)$ & $-40(1)$ & $0.0137(3)$ & $0.46(2)$ & 4 \\
\hline $\mathrm{B} 2334+61$ & 53615 & $20.579(1)$ & $41.35(3)$ & $-8.68(2)$ & $0.0075(1)$ & $0.92(1)$ & 5 \\
\hline
\end{tabular}

Refs.: 1. Lyne et al. $(2009) ; 2$. Livingstone et al. $(\underline{2010}) ; 3 . \overline{L i v i n g s t o n e}$ et al. $(2006) ; 4$. Shemar \& Lyne $(\overline{1996}) ; 5 . \overline{\text { Yuan et al. }}(\overline{2010})$

and glitches in the Vela pulsar (McCulloch et al. 1990; Alpar et al. 1993).

A comparison with PSR J1846-0258 is particularly interesting because its spin parameters $\left(\tau_{c}=0.8 \mathrm{kyr}\right.$ and a magnetic field of $\sim 4-5 \times 10^{13} \mathrm{G}$ ) are very similar to that of PSR J1119-6127. Until now, this rotationally powered radio quiet pulsar was the only very young pulsars for which a glitch of this magnitude had been detected. As observed in PSR J1119-6127, the large glitch on that pulsar presented a very efficient recovery, with $Q=9 \pm 3$, thanks to a large negative permanent frequency jump $\Delta \nu_{p}=-95 \pm 1 \mu \mathrm{Hz}$ (Livingstone et al. 2010). In addition to this anomalous recovery, the pulsar exhibited a radiative outburst episode in $\mathrm{X}$-rays resembling magnetar activity, and coincident with the large glitch (Gavriil et al. 2008; Kumar \& Safi-Harb 2008; Kuiper \& Hermsen 2009).

Glitches are believed to represent changes in the configuration of the star itself and in general are not associated to external factors. No changes on pulse shape or radiative behaviour have been reported for normal rotation powered pulsars, with the only exception being PSR J1846-0258. One glitch in the RRAT PSR J1819-1458 also exhibited an anomalous spin-down recovery, with $\Delta \dot{\nu}_{p}=0.79 \pm 1 \times$ $10^{-15} \mathrm{~Hz} \mathrm{~s}^{-1}$ and $Q^{\prime}=1.7 \pm 0.5$. Lvne et al. (2009) reported a possible increase in the pulsed emission rate accompanied by an increase of radio fluxes of the pulses associated with this glitch.

Despite the fact that these three neutron stars have shown anomalous glitch recoveries, they have somewhat different properties. Table 3 displays the values $\Delta \nu_{p}, \Delta \dot{\nu}_{p}, Q$ and $Q^{\prime}$ for the largest glitches in each of these three objects. Additionally, to compare with some other normal glitches, we also show the same parameters for the very large glitch in the young PSR B2334+61 (Yuan et al. 2010), a large glitch in the Vela-like pulsar PSR B1800-21 and a large glitch in the relatively old PSR B0355+54. Values were estimated from published fitted parameters and references are given in the last column. While the glitch in PSR J1119-6127 has both $Q$ and $Q^{\prime}$ greater than unity, the RRAT exhibits $Q^{\prime}>1$, but $Q<1$. On the other hand, the glitch in PSR J1846-0258 presents the opposite situation, with $Q>1$ but $Q^{\prime}<1$. The other two normal young pulsars present normal $Q<1$ and $Q^{\prime}<1$, owing to normal permanent jumps $\Delta \nu_{p}>0$ and $\Delta \dot{\nu}_{p}<0$.

The emission changes observed in PSR J1119-6127 following the 2007 glitch (Sect. 3] and 4) and the anomalous recovery the glitch presented are suggestive of a single con- nected event, as observed in PSR J1846-0258 and in RRAT PSR J1819-1458. Both these last objects are high magnetic field neutrons stars, just as PSR J1119-6127.

\subsubsection{Glitch models}

The most simple and intuitive model of a glitch is a starquake produced by sudden changes of the star shape (Baym et al. 1969; Ruderman 1969). These changes can be produced regularly as the star slows down and its shape evolves from an oblate configuration towards a more spherical one. However, the high glitch activity of some pulsars (like Vela or PSR B1737-30) cannot be accounted for by this model (McKenna \& Lyne 1990). A second model solved this problem by involving the physics of the interior of the star. In this model glitches are produced by a sudden transference of angular momentum from the inner neutron superfluid to the solid star crust. Any rotating superfluid slows down via migration away of the rotation axis of quantised vortices; carriers of the superfluid angular momentum. If the migration is stopped the superfluid retains its angular momentum and does not slow down. This model assumes that vortices often pin to different places of the inner crust due to interaction with the neutron superfluid associated with the crustal ion lattice (Alpar et al. 1984). In this way the inner superfluid keeps rotating at at higher rate and behaves as an angular momentum reservoir. The collective unpinning of many vortices may occur once stresses have built up due to the increasing differential rotation between the inner superfluid and the crust, producing the sudden spin up of the crust we observe as a glitch (Anderson \& Itoh 1975; Melatos \& Warszawski 2009).

Another model, proposed by Ruderman et al. (1998), states that vortex migration would push magnetic flux tubes away, producing stresses in the rigid crust that may be released through quake-like events. In addition to produce possible changes of the moment of inertia of the star, these reconfigurations could trigger the unpinning of a large number of vortices, producing a glitch.

Glitches in magnetars present a broad range of properties. Among them, the coincidence between radiative outburst and some glitches is the most relevant, but also the unusual recoveries and sometimes large post-glitch spin-down increments (Dib et al. 2008). These characteristics, which are not seen in normal radio pulsars, have induced the hypothesis that crust rearrangements caused by magnetic 
field decay or reconfigurations may trigger these glitches (Dib et al. 2007; Livingstone et al. 2010).

\subsubsection{Interpretation of glitch recoveries in terms of the pinning-unpinning model of glitches}

The pinning-unpinning model involves the existence of two main components: a resistive one, composed of all vortices which are continuously unpinning and pinning, and a capacitive one, containing those vortices that unpin only during a glitch (Alpar et al. 1996). The resistive component is continuously transferring angular momentum to the crust, as vortices unpin due to thermal fluctuations or quantum tunnelling (Alpar et al. 1984; Melatos \& Warszawski 2009). The capacitive component does not participate in the regular spin down of the star until a glitch occurs, and the star may contain several capacitive zones.

During a glitch the crust spins up and the rotational lag with the superfluid decreases, causing the effects of the resistive component to decrease (Wong et al. 2001). As a consequence, the spin-down rate increases due to the action of the always present external torque. This explains the initial spin-down jump at the glitch epoch and the following relaxation towards the equilibrium configuration, as vortices from the resistive component continue to pin and unpin.

Once the recovery is finished one would expect the star to follow the same spin-down rate as before the glitch. However, most young pulsars for which a recovery is observed present positive permanent jumps in frequency derivative. For the Crab pulsar, it has been proposed that this change in spin-down is caused by the creation of vortex trap zones, where many vortices pin and keep out of the regular slow down (Alpar et al. 1996; Wong et al. 2001). The pinning of a large number of vortices decreases the effective moment of inertia over which the external torque is acting, hence increasing the observed spin-down rate. In other words, during the glitch many vortices unpin, producing the observed spin up, but also a larger number of vortices pin in trap zones. In this context, $Q$ values less but similar to one are interpreted as a capacitive behaviour, where the pulsar is building an angular moment reservoir (Wong et al. 2001). Alpar et al. (1996) state that young pulsars like the Crab are still creating their capacitive components, which later will produce the larger glitch activity observed in slightly older pulsars like Vela. Additionally, they propose that the smaller and less frequent glitches in the Crab pulsar are likely to be triggered by starquakes, produced by the rapid spin-down of the star.

Following these ideas, and because of their similar age, the glitch activity in PSR J1119-6127 should present similar characteristics as the Crab. However, we have shown that the two last glitches of this pulsar present anomalous characteristics, specially on their spin-down rate recoveries. If the number of vortices that unpinned was considerably larger than the number of vortices that pinned (during the creation of capacitive zones), then the effective moment of inertia after the glitch would be larger than before, and the spin-down rate smaller. This would produce a postglitch spin-down evolution like the one observed in Fig. 10 What could cause this different behaviour is not clear, but the high magnetic field strength is something that PSRs J1119-6127, J1846-0258 and RRAT J1819-1458 have in common. Maybe, these glitches have been triggered by magnetic stresses, which could deform the crust, thereby changing the moment of inertia, or release pinned vortices. Either way, such a glitch would not be part of the regular glitch activity, and those processes following a normal glitch may not necessarily occur. An unusually large number of vortices may have unpinned in PSR J1119-6127 (considering the low glitch activity of pulsars of its age), producing a very large spin up. But, because the origin of the glitch was different, the unpinning of vortices overcame any possible vortex pinning, making up a larger final effective moment of inertia and therefore a smaller spin-down rate.

\subsection{Comparison with other neutron star populations}

\subsubsection{Comparison with other young pulsars}

The pulse profile of PSR J1119-6127 in its double-peaked mode (see middle panel of Fig. 5) has striking similarities with a group of young pulsars with a very distinct pulse shape. These energetic wide-beam pulsars, such as PSRs J1015-5719, J1105-6107 and J1420-6048, are characterized by their relatively wide double-peaked profile with steep inner edges and a high degree of linear polarization (Johnston \& Weisberg 2006). The similarity of this type of profiles with that of PSR J1119-6127 therefore suggests that the fiducial plane corresponds to the centre of the doublepeaked profile. Having the magnetic axis in the middle of the double-peaked profile makes it more natural that the two peaks have the same amplitude when they are visible. This scenario (case IV of the discussed geometries in Table 1) was also preferred after comparing the pulse profiles at the different observing frequencies.

The main difference between this pulsar and other pulsars of the same class of profiles might be that the PA curve is not as flat under the first of the two peaks in the doublepeaked mode. The estimated emission height of $\sim 500 \mathrm{~km}$ is somewhat larger than that of the other pulsars in this group (Johnston \& Weisberg 2006 derived emission heights of 380, 110 and $175 \mathrm{~km}$ for PSRs J1015-5719, J1105-6107 and J1420-6048 respectively). The relatively wide observed pulse profile is attributed to a relatively aligned geometry $\left(\alpha \simeq 17^{\circ}-30^{\circ}\right)$, or possibly smaller if only part of the open field line region is active.

There are a few young energetic pulsars which exhibit relatively strong individual pulses, although they are not glitch related. The strong pulses at the leading edge of the pulse profile of the Vela pulsar (Johnston et al. 2001) and at the trailing edge of PSR B1706-44 (Johnston \& Romani 2002) were dubbed "giant micro-pulses". These giant micropulses have large peak-flux densities, but their mean flux densities are not as extreme as the giant pulses observed for instance for the Crab pulsar (e.g. Lundgren et al. 1995). Unlike normal emission, the pulse-energy distribution of both giant pulses and giant micro-pulses are best described by a power law and are therefore thought to have a somewhat different origin. Unfortunately, not enough strong pulses were detected for PSR J1119-6127 to quantify their pulse energy distribution. Nevertheless, there are characteristics the strong pulses of PSR J1119-6127 do share with the giant micro-pulses: they all occur at the edges of the pulse profile 
and are broader than giant pulses (e.g. Hankins et al. 2003). Another characteristic that Vela and PSR B1706-44 have in common is that they are both detected at $\gamma$-ray energies (e.g. Abdo et al. 2010). The $\dot{E}$ of PSR J1119-6127 suggests that this pulsar might be a $\gamma$-ray source as well.

\subsubsection{Comparison with the RRATs}

It can be argued that if the line of sight to this pulsar were different, one would only be able to detect this pulsar via its individual pulses, hence it would have been classified as a RRAT. This would suggest that some of the RRATs are very similar in the sense that they may emit normal radio pulses, but not in the direction of Earth. There are more analogies one can draw between PSR J1119-6127 and the RRAT population. The unusual post-glitch over-relaxation observed for PSR J1119-6127 is not normal among the rest of the pulsar population, but it is similar to that observed for one of the RRATs (Lyne et al. 2009). Also, three of the seven RRATs with a measured $\dot{P}$ have a $B_{\mathrm{S}}>10^{13} \mathrm{G}$ (McLaughlin et al. 2009) and have therefore field strengths comparable with that of PSR J1119-6127. In addition it must be noted that the evolutionary track of PSR J1119-6127 in the $P-\dot{P}$ diagram may lead it towards the RRAT population rather than the bulk of the normal pulsars as is suggested by its braking index.

Having said this, a difference between PSR J1119-6127 and the RRATs is that in general there is no evidence that the event rate of RRAT pulses is time-dependent, certainly not at the level as observed for PSR J1119-6127. One of the RRATs is argued to have an increase in the RRAT-activity associated with a glitch (Lvne et al. 2009), although unlike PSR J1119-6127 it is always observed to be RRAT-like. If more sources like PSR J1119-6127 exist, there might be a population of neutron stars which emit pulses extremely sporadically after a glitch, possibly only some clustered pulses every so many years. This kind of sources, like "Lorimer burst" type of events (Lorimer et al. 2007), would be hard to detect. It will probably require next-generation telescopes (such as the LOFAR, ASKAP, MeerKAT or the SKA), which can afford long dwell times because of their large field-ofview, to discover this type of object.

PSR J1119-6127 shows erratic emission on both sides of the profile cone. This might be related to the general observation of the longitude resolved modulation index being higher at the edges of profile components (see e.g. Taylor et al. 1975; Weltevrede et al. 2006). This "edge effect" suggests that the locations of the profile peaks correspond to field lines at which the emission mechanism is stable. The edge effect is clearly observed for the Vela pulsar, PSR B1046-58 and B1706-44 (Johnston et al. 2001; Johnston \& Romani 2002), PSR B1133+16 (Kramer et al. 2003), and PSR B0656+14. The latter pulsar shows the strongest pulses in the centre of the profile (up to 116 times the average pulse intensity), but the emission on the leading edge can be up to a factor 2000 brighter than the average emission at those pulse longitudes (Weltevrede et al. 2006). Some of the RRATs might therefore be pulsars for which the line of sight only intersects this unstable part of the emission cone.

\subsubsection{Intermittent pulsars}

The double-peaked profile is only observed once in 12 years of data. This timescale is more similar to those of the intermittent pulsars (Lyne et al. 2010) than the shorter timescale mode changes observed in many other pulsars (e.g. Backer 1970; Rankin 1986). Apart from the similarity in timescale, the most obvious connection between PSR J1119-6127 and the intermittent pulsars is that changes in profile shape are linked to the timing properties of the star. However, the physical mechanism of this link are likely to be different. In the case of the intermittent pulsars it is believed that changes in the magnetosphere affect the pulsar rotation (the value of $\dot{\nu}$ ), while for PSR J1119-6127 we argue that it is possibly an external change in the magnetosphere that triggers the glitch. In any case, both the intermittent pulsars and PSR J1119-6127 show that there is an intimate connection between the neutron star interior and the magnetosphere.

\subsubsection{Comparison with PSR B0656+14}

PSR J1119-6127 shares an important feature with PSR B0656+14. Both pulsars were discovered using standard periodicity search techniques, but subsequently found to exhibit RRAT-like emission. Both pulsars therefore provide a link between the "normal" pulsars and the RRATlike pulsars. Pulsed X-rays have been observed for both PSR J1119-6127 (Gonzalez et al. 2005) and PSR B0656+14 (Córdova et al. 1989; De Luca et al. 2005). In the radio band they both have a more or less triangular-shaped pulse profile which covers only a part of the open field line region (in the case of PSR B0656+14 there is a trailing shoulder, while PSR J1119-6127 has the intermittent trailing peak). They both have a high degree of linear polarization at 1.4 $\mathrm{GHz}$ and it will be interesting to see if PSR J1119-6127 becomes depolarized at higher frequencies, like PSR B0656+14 (Johnston et al. 2006).

There are also some important differences between PSRs J1119-6127 and B0656+14. PSR B0656+14 is a middle-aged pulsar located near the bulk of pulsars in the $P$ $\dot{P}$ diagram, while PSR J1119-6127 is very young and has an extremely strong magnetic field. The RRAT-like emission of PSR J1119-6127 is found in an separate profile component (more like giant micro-pulses), while the strongest pulses of PSR B0656+14 are found at the same pulse longitude as the normal emission. Therefore the RRAT-like emission of PSR B0656+14 is interpreted as being the tail of an extended pulse-energy distribution (Weltevrede et al. 2006), while in the case of PSR J1119-6127 and the giant micro-pulses the RRAT-like emission is more distinct from the normal emission. Also, the emission of PSR B0656+14 was found to be more extreme at low observing frequencies, while for PSR J1119-6127 strong pulses possibly occur more often at higher observing frequencies. Therefore the physics of the strong pulses observed for PSR B0656+14 might be more related to those observed for older pulsars at very low frequencies (e.g. Ershov \& Kuzmin 2005). In addition to these differences the strong pulses of PSR B0656+14 are not related to glitches. 


\subsubsection{Comparison with magnetars}

Because of the high magnetic field strength of PSR J11196127 there is an obvious direct link with the magnetars. Links between magnetars and rotation-powered pulsars have been suggested before as for instance PSR J1846-0258, a young (radio-quiet) pulsar with a similar magnetic field strength to that of PSR J1119-6127, has shown magnetarlike X-ray bursts Gavriil et al. 2008; Kumar \& Safi-Harb 2008). In addition, an over-recovery $(Q=8.7 \pm 2.5$; Livingstone et al. 2010) has been claimed for that source, as well as for magnetar AXP $4 \mathrm{U} 0142+61(Q=1.07 \pm 0.02$; Gavriil et al. 2009).

Three of the magnetars are detected as radio pulsars (Camilo et al. 2006, 2007; Levin et al. 2010) and they show characteristics similar to PSRs J1119-6127 and B0656+14. In particular all three have a high degree of linear polarization (Camilo et al. 2007; Kramer et al. 2007; Camilo et al. 2008) and individual pulses from PSR J1810 197 (Servlak et al. 2009) and J1623-4950 (Levin et al. 2010) show spiky emission similar to that seen in PSR B0656+14 (Weltevrede et al. 2006). Curiously, evidence seems to suggest that the magnetars are close to being aligned rotators (Camilo et al. 2007, 2008) and this might also be the case for PSR 1119-6127 and possibly PSR B0656+14 (Everett \& Weisberg 2001). Could it be that the aligned geometry is responsible for some of these attributes? We note also that the $\gamma$-ray profile of PSR B0656+14 is peculiar (Weltevrede et al. 2010) and we await the detection of PSR J1119-6127 with the Fermi satellite with interest.

\subsection{What are RRATs?}

The inconsistency between the observed supernova rate and the size of the RRAT population suggests there are links between the different neutron star classes Keane \& Kramer 2008). Indeed, it has been argued that one of the RRATs, PSR J1819-1458, might be evolved from the magnetars based on the unusual $\dot{P}$ change after its glitch (Lyne et al. 2009). Because of the movement of the Crab pulsar in the $P-\dot{P}$ diagram the young pulsars and the magnetars appear to be linked as well (Lvne 2004). The view that the RRATs are an evolutionary stage rather than a separate class of neutron stars is further strengthened by the discovery of "missing links": pulsars which can be classified both as pulsars or as RRATs. Some RRATs will turn out to be pulsars with extended pulse energy distributions (like that of PSR B0656+14; Weltevrede et al. 2006), others might have a "normal" profile component (like PSR J1119-6127) which happen to be missed by our line of sight, others might be pulsars with extreme null lengths (see e.g. Burke-Spolaor \& Bailes 2010) and some might be magnetars with more extreme spiky radio emission than XTE J1810197 (Serylak et al. 2009).

With the above in mind, a valid question to ask oneself is what you are left with when you remove the pulsars that fall in the above categories from the RRAT population. Will there be any "true" RRATs left? Or are RRATs simply the combination of the extreme ends of the other classes of neutron stars? Because RRATs do not form a separate island in the $P-\dot{P}$ diagram and because it proves to be difficult to define the RRATs based on their physical prop- erties (see e.g. Keane et al. 2010), it is tempting to apply Occam's razor and assume that the RRATs do not form a distinct population of pulsars.

A problem with the term RRAT is that it is not clearly defined. The definition that RRATs are pulsars which are not detectable via periodicity searches is insufficient because (as illustrated by PSR B0656+14) it depends on the distance of the source (and the integration time of the observation and the sensitivity of the telescope). By accepting that RRATs are not a separate class of pulsars the question of how to define a RRAT is irrelevant. More useful would be to define how erratic (hence "RRAT-like") the emission of a pulsar is. Such a definition would allow a better investigation of how this type of emission is correlated with other neutron star parameters.

The degree of RRAT-like behaviour can be quantified by intermittency ratio (McLaughlin \& Cordes 2003; Deneva et al. 2009; Keane et al. 2010), which compares the signal-to-noise ratio of the detection of the pulsar in a single-pulse search with that of a periodicity search, or the R-parameter (Johnston et al. 2001), which is a normalized measure of the brightest observed pulse. Both measures have the problem that it depends on both the sensitivity of the telescope and the duration of the observation. A straightforward way to define the degree of RRAT-like behaviour would be to use the modulation index (Eq. 1), which quantifies the broadness of an amplitude distribution and is observing-system independent. Note that $m$ is usually calculated as a function of pulse longitude. This makes a big difference for especially the magnetar emission, which shows many bursts per stellar rotation.

For RRATs $\langle I\rangle$ is below the detection threshold and therefore the modulation index cannot be calculated (the same problem applies for the intermittency ratio or the Rparameter). Nevertheless one can determine a lower limit on the modulation index by taking zero intensity for the undetected pulses and replacing $\langle I\rangle$ by its maximum allowed value, which is the detection threshold for the average pulse intensity $\langle I\rangle_{\text {tresh }}$. The resulting limit is

$m \geqslant\left(\frac{1}{N_{\text {tot }}} \sum_{i=1}^{N_{\text {det }}}\left(I_{i} /\langle I\rangle_{\text {tresh }}-1\right)^{2}\right)^{1 / 2}$,

where $N_{\text {det }}$ is the number of detected pulses.

The modulation index for selected sources can be found in Table 4. One can see that the modulation index of the normal emission of PSR J1119-6127 is typical for that of radio pulsars. Unfortunately, the lower limit of the modulation index of the RRAT-like emission of PSR J1119-6127 is not much higher than that of the normal emission, and therefore it is consistent with all types of emission. To improve this limit one needs to use an instrument which is much more sensitive than Parkes, such as the SKA. On the other hand, one can see that the modulation index of RRAT J1819-1458 must be much higher than that of normal pulsar emission and is possibly more similar to that found for magnetar emission or that of giant pulses. Measurements of $m$ for more RRATs and other sources with extreme radio emission has the potential to differentiate between different types of radio emission. 
Table 4. Table with some typical values of the modulation index $m$ measured for different types of emission.

\begin{tabular}{|c|c|c|c|}
\hline Source & Type of emission & $m$ & Ref. \\
\hline Typical pulsar & Normal & 0.5 & $a$ \\
\hline Crab pulsar & Giant pulses & $5-8$ & \\
\hline PSR B0656+14 & RRAT-like & $\sim 5$ & $c$ \\
\hline PSR J1119-6127 & Normal & $\sim 0.5$ & $d$ \\
\hline PSR J1119-6127 & RRAT-like & $\geqslant 0.6$ & $e$ \\
\hline XTE J1810-197 & Magnetar emission & $1-10$ & $f$ \\
\hline PSR J1819-1458 & RRAT & $\geqslant 6.6$ & $g$ \\
\hline
\end{tabular}

a Typical minimum value of the longitude resolved modulation index at a wavelength around $21 \mathrm{~cm}$ (Weltevrede et al. 2006) and $m$ is typically observed to flare up at the profile edges.

$b$ Longitude resolved modulation index at the position of the giant pulses at $1400 \mathrm{MHz}$ (Weltevrede et al. 2006). The actual value might be higher if the giant pulses were not entirely resolved.

$c$ Measured by Weltevrede et al. (2006) at a frequency of 327 $\mathrm{MHz}$ in the centre of the profile (where the brightest burst occurred).

$d$ This paper. The modulation index flares up at the profile edges. $e$ Modulation index of the RRAT-like component based on the single 20 -cm observation with the four individual pulses.

$f$ The longitude resolved modulation index is variable in time, but in the range of $m=1-4$ at $1.4 \mathrm{GHz}$ or $m=1-10$ at $8.35 \mathrm{GHz}$ (Servlak et al. 2009).

$g$ This is the RRAT with the best determined pulse energy distribution. The estimated limit is based on this distribution (Supplementary Figure 2 of McLaughlin et al. 2006) in combination with the quoted event rate of 229 detected pulses in 13 hours of data and the statement that average peak flux density must be less than $0.05 \%$ that of the strongest detected burst.

\section{CONCLUSIONS}

We found that the young high-magnetic field pulsar J11196127 exhibits three types of pulsar behaviour. It has an "intermittent" profile peak trailing the peak associated with "normal" emission and these profile components are flanked by two much more erratic components, which can be argued to be "RRAT-like". Both the erratic components and the intermittent component are observed around the same epoch and appear to be transient events. It can therefore not be ruled out that they represent different phases of the on- and off-switching process, possibly related to regions on the polar cap which usually fail in producing the positron-electron pairs required for the emission mechanism to work. Nevertheless the overall profile symmetry suggests that the more steady components are located in the centre of the beam and the more flickering components at the edges. This is consistent with the "edge effect", which states that in general the emission is more erratic at the edges of the profile components. Some of the RRATs might therefore be pulsars for which the line-of-sight misses the part of the beam with more steady emission.

Both the intermittent and RRAT-like events are very rare and are preceded by a large amplitude glitch that occurred directly before these phenomenon were observed. This is the first time that a glitch, or the post-glitch recovery process, is observed to influence the radio emission process of a normal (non-RRAT) pulsar. The glitch was followed by a post-glitch behaviour which is very unusual for the pulsar population as a whole, but it is similar to that observed for one of the RRATs (PSR J1819-1458). After the glitch, the spin-down rate relaxed to a smaller value than the projected pre-glitch value. We interpret this as an increase of the effective moment of inertia produced by an excessive number of unpinning vortices. By considering this anomaly and the emission changes observed, we believe that the glitch may have been triggered by magnetic reconfigurations.

Although we measure a braking index $n=2.684 \pm 0.002$, glitches like the one reported here make the effective braking index larger. Nevertheless, the evolutionary track of PSR J1119-6127 in the $P-\dot{P}$ diagram may lead it towards the RRAT population rather than the bulk of the normal pulsars. The occurrence-rate of RRAT-like pulses must have been higher around the glitch epoch (as is claimed for J1819-1458), suggesting a link with the glitch-recovery process. More precise, the RRAT-like pulses were observed during the initial fast recovery (on a timescale of $\sim 21$ days) rather than the later slow recovery (on a timescale of $\sim 208$ days). This allows the possibility that RRAT-like activity is in general low for young, high-magnetic field pulsars (except after a glitch) and increases when pulsars age.

Like PSR B0656+14, which would be classified as a RRAT were it more distant, PSR J1119-6127 provides a link between the known neutron star population and the RRATs. Additional links could be other young pulsars which show "giant micro-pulses", the magnetar population which show PSR B0656+14-like spiky emission and pulsars with extreme long null lengths. It appears that RRATs represents a corner in the $P-\dot{P}$ diagram in which the pulsars have the most erratic emission. We argue that the modulation index is a good measure of how RRAT-like the emission is. The modulation quantifies the broadness of the pulse energy distribution and is (unlike for instance the "intermittency ratio" or the "R-parameter") independent of the sensitivity of the telescope or observation duration. For RRATs (which are not detected in a periodicity search) one can only derive a lower limit. This limit is estimated to be $m \geqslant 6.6$ for the RRAT PSR J1819-1458, which although high for pulsar standards is not necessarily higher than that observed for magnetars. Additional observations could place stronger limits on how high the modulation index of RRAT emission is and can potentially be used to distinguish between the different sub-classes of the RRATs.

Not only are the strong individual pulses of PSR J11196127 reminiscent of those of other young pulsars, but so is the shape of its pulse profile in its double-peaked mode. The similarity with other "energetic wide-beam pulsar" suggests that the magnetic axis is located in between the two components. The derived emission height for that scenario is $\sim 500 \mathrm{~km}$, somewhat higher than that derived for other young pulsars. thereby suggesting a relatively aligned geometry $\left(\alpha \sim 17^{\circ}-30^{\circ}\right)$, or slightly more aligned if only part of the open field line region is ever active.

\section{ACKNOWLEDGMENTS}

The authors want to thank Ben Stappers and Evan Keane for the useful discussions. The Australia Telescope is funded by the Commonwealth of Australia for operation as a National Facility managed by the CSIRO. 
The glitch-induced identity changes of PSR J1119-6127

\section{REFERENCES}

Abdo A. A., et al. 2010, Astrophys. J. Supp. Series, 187, 460

Alpar M. A., Anderson P. W., Pines D., Shaham J., 1984, ApJ, 276, 325

Alpar M. A., Chau H. F., Cheng K. S., Pines D., 1993, ApJ, 409, 345

Alpar M. A., Chau H. F., Cheng K. S., Pines D., 1996, ApJ, 459, 706

Alpar M. A., Pines D., Anderson P. W., Shaham J., 1984, ApJ, 276, 325

Anderson P. W., Itoh N., 1975, Nature, 256, 25

Backer D. C., 1970, Nature, 228, 1297

Baring M. G., Harding A. K., 1998, ApJ, 507, L55

Baym G., Pethick C., Pines D., Ruderman M., 1969, Nature, 224, 872

Blandford R. D., Romani R. W., 1988, MNRAS, 234, 57P

Blaskiewicz M., Cordes J. M., Wasserman I., 1991, ApJ, 370,643

Burke-Spolaor S., Bailes M., 2010, MNRAS, 402, 855

Camilo F., Kaspi V. M., Lyne A. G., Manchester R. N., Bell J. F., D'Amico N., McKay N. P. F., Crawford F., 2000, ApJ, 541, 367

Camilo F., Ransom S. M., Halpern J. P., Reynolds J., 2007, ApJ, 666, L93

Camilo F., Ransom S. M., Halpern J. P., Reynolds J., Helfand D. J., Zimmerman N., Sarkissian J., 2006, Nature, 442, 892

Camilo F., Reynolds J., Johnston S., Halpern J. P., Ransom S. M., 2008, ApJ, 679, 681

Camilo F., Reynolds J., Johnston S., Halpern J. P., Ransom S. M., van Straten W., 2007, ApJ, 659, L37

Cordes J. M., Shannon R. M., 2008, ApJ, 682, 1152

Córdova F. A., Hjellming R. M., Mason K. O., Middleditch

J., 1989, ApJ, 345, 451

Crawford F., Gaensler B. M., Kaspi V. M., Manchester R. N., Camilo F., Lyne A. G., Pivovaroff M. J., 2001, ApJ, 554, 152

De Luca A., Caraveo P. A., Mereghetti S., Negroni M., Bignami G. F., 2005, ApJ, 623, 1051

Deneva J. S., et al. 2009, ApJ, 703, 2259

Dib R., Kaspi V. M., Gavriil F. P., 2007, Astrophys. Space

Sci., p. 44

Dib R., Kaspi V. M., Gavriil F. P., 2008, ApJ, 673, 1044

Edwards R. T., Hobbs G. B., Manchester R. N., 2006, MNRAS, 372, 1549

Edwards R. T., Stappers B. W., 2002, A\&A, 393, 733

Ershov A. A., Kuzmin A. D., 2005, A\&A, 443, 593

Everett J. E., Weisberg J. M., 2001, ApJ, 553, 341

Gaensler B. M., McLaughlin M., Reynolds S., Borkowski K., Rea N., Possenti A., Israel G., Burgay M., Camilo F., Chatterjee S., Kramer M., Lyne A., Stairs I., 2007, Ap\&SS, 308, 95

Gavriil F. P., Dib R., Kaspi V. M., 2009, ArXiv e-prints (astro-ph/0905.1256)

Gavriil F. P., Gonzalez M. E., Gotthelf E. V., Kaspi V. M., Livingstone M. A., Woods P. M., 2008, Science, 319, 1802

Gil J. A., Gronkowski P., Rudnicki W., 1984, A\&A, 132, 312

Gonzalez M. E., Kaspi V. M., Camilo F., Gaensler B. M., Pivovaroff M. J., 2005, ApJ, 630, 489
Hankins T. H., Kern J. S., Weatherall J. C., Eilek J. A., 2003, Nature, 422

Hessels J. W. T., Ransom S. M., Kaspi V. M., Roberts M. S. E., Champion D. J., Stappers B. W., 2008, in C. Bassa, Z. Wang, A. Cumming, \& V. M. Kaspi ed., 40 Years of Pulsars: Millisecond Pulsars, Magnetars and More Vol. 983 of American Institute of Physics Conference Series, The GBT350 Survey of the Northern Galactic Plane for Radio Pulsars and Transients. pp 613-615

Hobbs G. B., Edwards R. T., Manchester R. N., 2006, MNRAS, 369, 655

Johnston S., Karastergiou A., Willett K., 2006, MNRAS, 369,1916

Johnston S., Romani R., 2002, MNRAS, 332, 109

Johnston S., van Straten W., Kramer M., Bailes M., 2001, ApJ, 549, L101

Johnston S., Weisberg J. M., 2006, MNRAS, 368, 1856

Kaplan D. L., 2008, in Y.-F. Yuan, X.-D. Li, \& D. Lai ed., Astrophysics of Compact Objects Vol. 968 of American Institute of Physics Conference Series, Nearby, Thermally Emitting Neutron Stars. pp 129-136

Karastergiou A., Hotan A. W., van Straten W., McLaughlin M. A., Ord S. M., 2009, MNRAS, 396, L95

Keane E. F., Kramer M., 2008, MNRAS, 391, 2009

Keane E. F., Ludovici D. A., Eatough R. P., Kramer M., Lyne A. G., McLaughlin M. A., Stappers B. W., 2010, MNRAS, 401, 1057

Kramer M., Karastergiou A., Gupta Y., Johnston S., Bhat N. D. R., Lyne A. G., 2003, A\&A, 407, 655

Kramer M., Lyne A. G., O'Brien J. T., Jordan C. A., Lorimer D. R., 2006, Science, 312, 549

Kramer M., Stappers B. W., Jessner A., Lyne A. G., Jordan C. A., 2007, MNRAS, 377, 107

Kuiper L., Hermsen W., 2009, A\&A, 501, 1031

Kumar H. S., Safi-Harb S., 2008, ApJ, 678, L43

Levin L., Bailes M., Bates S., Bhat N. D. R., Burgay M., Burke-Spolaor S., D'Amico N., Johnston S., Keith M., Kramer M., Milia S., Possenti A., Rea N., Stappers B., van Straten W., 2010, ApJ, 721, L33

Livingstone M. A., Kaspi V. M., Gavriil F. P., 2010, ApJ, 710,1710

Livingstone M. A., Kaspi V. M., Gavriil F. P., Manchester R. N., Gotthelf E. V. G., Kuiper L., 2007, Astrophys. Space Sci., 308, 317

Livingstone M. A., Kaspi V. M., Gotthelf E. V., Kuiper L., 2006, ApJ, 647, 1286

Lorimer D. R., Bailes M., McLaughlin M. A., Narkevic D. J., Crawford F., 2007, Science, 318, 777

Lorimer D. R., Kramer M., 2005, Handbook of Pulsar Astronomy. Cambridge University Press

Lundgren S. C., Cordes J. M., Ulmer M., Matz S. M., Lomatch S., Foster R. S., Hankins T., 1995, ApJ, 453, 433

Luo Q., Melrose D., 2007, MNRAS, 378, 1481

Lyne A., Hobbs G., Kramer M., Stairs I., Stappers B., 2010, Science, 329, 408

Lyne A. G., 2004, in F. Camilo \& B. M. Gaensler ed., Young Neutron Stars and Their Environments Vol. 218 of IAU Symposium, From Crab Pulsar to Magnetar?. pp 257-+

Lyne A. G., McLaughlin M. A., Keane E. F., Kramer M., Espinoza C. M., Stappers B. W., Palliyaguru N. T., Miller J., 2009, MNRAS, 400, 1439

Lyne A. G., Shemar S. L., Graham-Smith F., 2000, MN- 
RAS, 315, 534

McCulloch P. M., Hamilton P. A., McConnell D., King

E. A., 1990, Nature, 346, 822

McKenna J., Lyne A. G., 1990, Nature, 343, 349

Manchester R. N., et al. 2001, MNRAS, 328, 17

McLaughlin M. A., Cordes J. M., 2003, ApJ, 596, 982

McLaughlin M. A., et al. 2006, Nature, 439, 817

McLaughlin M. A., Lyne A. G., Keane E. F., Kramer M., Miller J. J., Lorimer D. R., Manchester R. N., Camilo F., Stairs I. H., 2009, MNRAS, 400, 1431

McLaughlin M. A., Rea N., Gaensler B. M., Chatterjee S., Camilo F., Kramer M., Lorimer D. R., Lyne A. G., Israel G. L., Possenti A., 2007, ApJ, 670, 1307

Melatos A., Warszawski L., 2009, ApJ, 700, 1524

Press W. H., Teukolsky S. A., Vetterling W. T., Flannery B. P., 1992, Numerical recipes in C. The art of scientific computing. Cambridge: University Press, —c1992, 2nd ed.

Radhakrishnan V., Cooke D. J., 1969, Astrophys. Lett., 3, 225

Radhakrishnan V., Manchester R. N., 1969, Nature, 222, 228

Rankin J. M., 1986, ApJ, 301, 901

Reynolds S. P., Borkowski K. J., Gaensler B. M., Rea N., McLaughlin M., Possenti A., Israel G., Burgay M., Camilo F., Chatterjee S., Kramer M., Lyne A., Stairs I., 2006, ApJ, 639, L71

Ruderman M., 1969, Nature, 223, 597

Ruderman M., Zhu T., Chen K., 1998, ApJ, 492, 267

Serylak M., Stappers B. W., Weltevrede P., Kramer M., Jessner A., Lyne A. G., Jordan C. A., Lazaridis K., Zensus J. A., 2009, MNRAS, 394, 295

Shemar S. L., Lyne A. G., 1996, MNRAS, 282, 677

Smith D. A., et al. 2008, A\&A, 492, 923

Standish E. M., 1998, A\&A, 336, 381

Taylor J. H., Manchester R. N., Huguenin G. R., 1975, ApJ, 195, 513

von Hoensbroech A., Lesch H., Kunzl T., 1998, A\&A, 336, 209

Wang N., Manchester R. N., Johnston S., 2007, MNRAS, 377, 1383

Wang N., Manchester R. N., Pace R., Bailes M., Kaspi V. M., Stappers B. W., Lyne A. G., 2000, MNRAS, 317, 843

Weltevrede P., Edwards R. T., Stappers B. W., 2006, A\&A, 445,243

Weltevrede P., et al. 2010, ApJ, 708, 1426

Weltevrede P., Johnston S., 2008, MNRAS, 391, 1210

Weltevrede P., Johnston S., Manchester R. N., Bhat R., Burgay M., Champion D., Hobbs G. B., Kizıltan B., Keith M., Possenti A., Reynolds J. E., Watters K., 2010, PASA, 27, 64

Weltevrede P., Stappers B. W., Rankin J. M., Wright G. A. E., 2006, ApJ, 645, L149

Weltevrede P., Wright G. A. E., Stappers B. W., Rankin J. M., 2006, A\&A, 458, 269

Wong T., Backer D. C., Lyne A., 2001, ApJ, 548, 447

Woods P. M., Thompson C., 2006, Compact Stellar X-ray Sources. Cambridge University Press

Yuan J. P., Manchester R. N., Wang N., Zhou X., Liu Z. Y., Gao Z. F., 2010, ApJ, 719, L111 